ARTICLE

DOI: $10.1038 / s 41467-017-01169-0$

\title{
Dynamic regulation of canonical TGF $\beta$ signalling by endothelial transcription factor ERG protects from liver fibrogenesis
}

Neil P. Dufton ${ }^{1}$, Claire R. Peghaire ${ }^{1}$, Lourdes Osuna-Almagro ${ }^{1}, C^{2}$ Claudio Raimondi ${ }^{1}, V^{\prime k t o r i a ~ K a l n a}{ }^{1}$, Abhishek Chauhan (1) 2, Gwilym Webb (1) 2, Youwen Yang ${ }^{1}$, Graeme M. Birdsey (1) ${ }^{1}$, Patricia Lalor ${ }^{2}$, Justin C. Mason ${ }^{1}$, David H. Adams ${ }^{2} \&$ Anna M. Randi ${ }^{1}$

The role of the endothelium in protecting from chronic liver disease and TGF $\beta$-mediated fibrosis remains unclear. Here we describe how the endothelial transcription factor ETS-related gene (ERG) promotes liver homoeostasis by controlling canonical TGF $\beta$-SMAD signalling, driving the SMAD1 pathway while repressing SMAD3 activity. Molecular analysis shows that ERG binds to SMAD3, restricting its access to DNA. Ablation of ERG expression results in endothelial-to-mesenchymal transition (EndMT) and spontaneous liver fibrogenesis in EC-specific constitutive hemi-deficient ( $\mathrm{Frg}^{\mathrm{CEC}-\mathrm{Het}}$ ) and inducible homozygous deficient mice $\left(E r{ }^{i E C-K O}\right)$, in a SMAD3-dependent manner. Acute administration of the TNF- $\alpha$ inhibitor etanercept inhibits carbon tetrachloride $\left(\mathrm{CCL}_{4}\right)$-induced fibrogenesis in an ERG-dependent manner in mice. Decreased ERG expression also correlates with EndMT in tissues from patients with end-stage liver fibrosis. These studies identify a pathogenic mechanism where loss of ERG causes endothelial-dependent liver fibrogenesis via regulation of SMAD2/3. Moreover, ERG represents a promising candidate biomarker for assessing EndMT in liver disease.

\footnotetext{
${ }^{1}$ Vascular Sciences, Imperial Centre for Translational and Experimental Medicine, National Heart and Lung Institute, Imperial College London, London W12 ONN, UK. ${ }^{2}$ Centre for Liver Research, Institute of Biomedical Research, Institute of Immunology and Immunotherapy, College of Medical and Dental Sciences, University of Birmingham, Birmingham B15 2TT, UK. Correspondence and requests for materials should be addressed to A.M.R. (email: a.randi@imperial.ac.uk)
} 
C hronic liver disease (CLD) is an increasing global health burden; in the UK, liver disease is the fifth biggest cause of mortality with rates doubling from 1991 to $2007^{1}$. CLD can be triggered by numerous factors including diet, alcohol, viral infection and genetic disorders, which share common features including excessive inflammation ${ }^{2}$, dysregulated transforming growth factor (TGF $\beta$ ) signalling ${ }^{3}$ and dramatic disruption in the vascular architecture of the liver ${ }^{4,5}$. Recent studies have revealed that disruption of endothelial cell (EC) homoeostasis can initiate tissue damage and fibrosis ${ }^{6-8}$. Notably, EC have been shown to lose their lineage-specific markers and morphology and acquire a mesenchymal-like phenotype in a process termed endothelial-tomesenchymal transition (EndMT) $)^{9}$. EndMT is associated with human pathologies such as early vein-graft rejection ${ }^{10}$ and atherosclerosis ${ }^{11}$, where it correlates with disease severity. EndMT has been shown to be induced by TGF $\beta$ in vitro and in vivo ${ }^{10,12}$, and to be enhanced by inflammatory mediators including TNF- $\alpha^{13}, 14$.

Canonical TGF $\beta /$ bone morphogenetic protein (BMP) signalling can activate two opposing signalling cascades, maintaining homoeostasis via phosphorylation of transcription factors SMAD1/5/8, while instigating pro-fibrotic signalling via phosphorylation of SMAD2/3 ${ }^{15}$ (Supplementary Fig. 1). In EC, TGF $\beta$ isoforms activate SMAD1/5/8 signalling via the receptor ACVRL1 and its co-factor endoglin $(\mathrm{ENG})^{16}$. BMP ligand-BMP receptor (BMPR) and TGF $\beta$-ACVRL1 interactions selectively induce SMAD1 phosphorylation and directly inhibit TGF $\beta$-ALK5SMAD3-mediated transcription ${ }^{17}$. Importantly, TGF- $\beta$-induced signalling is influenced by cross-talk with multiple pathways and by lineage-specific co-factors ${ }^{18}$. Thus, regulation of the balance between SMAD1 and SMAD3 signalling is crucial in maintaining EC homeostasis ${ }^{19,20}$.

The ETS transcription factor family plays important roles in vascular development and angiogenesis ${ }^{21}$. The ETS-related gene (ERG) is the most abundant ETS factor in adult ECs and is essential for endothelial lineage identity; indeed, it is one of only three transcription factors required for reprogramming of progenitors to endothelium ${ }^{22}$. ERG is crucial for embryonic development and vascular stability ${ }^{23,24}$, angiogenesis ${ }^{25}$ and protection from vascular inflammation ${ }^{26,27}$. ERG exerts its pro-homoeostatic, anti-inflammatory function in ECs by driving expression of homoeostatic genes while repressing pro-inflammatory gene expression and inhibiting cytokine-induced EC activation ${ }^{21,28}$. In turn, pro-inflammatory molecules TNF- $\alpha^{26}$ and lipopolysaccharides (LPS $)^{27}$ induce a significant loss in ERG expression in ECs, suggesting that regulation of ERG expression is key to control of the balance between endothelial homoeostasis and inflammatory signalling.

In this study, we show that ERG maintains the homoeostatic balance of SMAD-dependent signalling in the endothelium, by promoting the SMAD1 pathway while repressing SMAD2/3 activity. We show that loss of ERG in vitro and in vivo results in spontaneous EndMT which is dependent on enhanced SMAD2/3 activity. TNF- $\alpha$ blockade protects ERG expression and reduces phosphorylation of SMAD2/3 in an acute model of liver fibrosis, but is ineffective in ERG hemi-deficient mice. Finally, we show that ERG expression is lost in liver EC from cirrhotic patients with fibrosis related to alcoholic liver disease (ALD) or primary biliary cirrhosis (PBC) and inversely correlates with increased markers of EndMT. Therefore, this study identifies a central role for ERG in regulating EC canonical TGF $\beta$-SMAD signalling to prevent EndMT and ultimately tissue fibrogenesis. Loss of endothelial ERG expression is an early, causative event during tissue fibrogenesis, linked to inflammatory pathways, which can be targeted therapeutically.

\section{Results}

ERG regulates SMAD signalling in EC and protects from EndMT. Gene ontology analysis of transcriptome data from ERG-deficient human umbilical cord endothelial cells (HUVEC) ${ }^{25}$ identified 41 target genes associated with TGF $\beta / B M P$ canonical signalling as putative ERG targets (Supplementary Table 1; Fig. 1a). qRT-PCR validation of selected hits and other relevant genes is show in Fig. 1b. Inhibition of ERG in HUVEC caused a reduction in gene expression of SMAD1, ACVRL1 (ALK1), endoglin (ENG) and inhibitor of DNA binding 1 (ID1). This response was mirrored by elevated expression of TGF $\beta$ isoforms 1 and 2 and of mesenchymal genes collagen A1 (Col1A1), smooth muscle actin (SMA) and smooth muscle calponin (CNN1). Gene expression levels of other signalling SMADs (5 and 8) were not significantly changed in ERG-deficient cells (Supplementary Fig. 2A). A second siRNA (\#2) targeting ERG generated a similar mRNA profile (Supplementary Fig. 2B). Protein levels of SMAD1 and its associated proteins, ENG and ID1, were also significantly reduced (Fig. 1c, d and Supplementary Fig. 2C). Loss of SMAD1 expression was confirmed in vivo, within liver tissue from EC-specific constitutive Erg hemideficient mice ${ }^{23}$ (Erg ${ }^{C E C-H e t}$; Fig. 1e; arrows). Gene and protein expression levels of SMAD3 and its receptor TGFBR1/ALK5 were unaffected (Fig. 1b-d). However, there was increased expression of the downstream targets SMA (Fig. 1d) and TGF $\beta 2$ (Fig. 1f). Furthermore, immunofluorescence microscopy revealed increased TGF $\beta 2$ expression in ERG-deficient EC in vitro (Fig. 1g) and in large blood vessels in the liver of $\mathrm{Erg}^{\mathrm{CEC}-\mathrm{Het}}$ mice in vivo (Fig. 1h; arrows). The profile of ERG-deficient cells, namely marked upregulation of mesenchymal markers and downregulation of endothelial lineage identity markers, together with transition from the typical rounded/cobblestone morphology to a distinct mesenchymal-like shape $\mathrm{e}^{25}$, suggest that the cells are undergoing EndMT. Thus, ERG reciprocally regulates TGF $\beta /$ BMP canonical signalling in EC, by driving the SMAD1 pathway while repressing the SMAD2/3 pathway to protect EC from EndMT.

ERG promotes SMAD1 whilst repressing SMAD2/3 signalling in EC. To assess whether ERG inversely regulates SMAD1 and SMAD2/3 transcriptional activity, we used a SMAD1-dependent BMP Response Element luciferase reporter (BRE reporter) ${ }^{29}$ and a SMAD2/3-dependent SMAD-Binding Element luciferase reporter (SBE reporter) ${ }^{30}$. The SMAD2/3 activator TGF $\beta 2$ induced enhanced transactivation activity of the SBE reporter (Supplementary Fig. 3A), with comparable effects at 1 and $10 \mathrm{ng}$ $\mathrm{ml}^{-1}$ TGF $\beta 2$. All following experiments were carried out with $10 \mathrm{ng} \mathrm{ml}^{-1}$ TGF $\beta 2$, in line with previous in vitro studies on EndMT $^{31,32}$. Ligand specificity of BMP9 $\left(1 \mathrm{ng} \mathrm{ml}^{-1}\right)$ and TGF $\beta 2$ $\left(10 \mathrm{ng} \mathrm{ml}^{-1}\right)$ was shown by selective induction of their target genes ENG and CNN1, respectively (Supplementary Fig. 3B). In control siRNA transfected cells, treatment with BMP9-induced a robust BRE-, but no SBE-mediated signal, whilst TGF $\beta 2$ induced the opposite response, as expected (Fig. 2a, b). Inhibition of ERG expression caused a significant reduction in SMAD1-dependent BRE transactivation following BMP9 treatment (Fig. 2a); this was paralleled by decreased phosphorylation of SMAD1 in response to BMP9 (Supplementary Fig. 3C) and TGF $\beta 2$ (Supplementary Fig. 3D; quantified Supplementary Fig. 3E), suggesting that ERG regulates SMAD1 levels and activity. Notably, ERG overexpression did not further enhance BMP9-induced BRE reporter transactivation activity (Fig. 2c). TGF $\beta 2$ induced a modest increase in SBE reporter transactivation, which was significantly enhanced following the deletion of ERG by siRNA (Fig. 2b). Conversely, ERG-overexpression completely inhibited TGF $\beta 2$ - 
induced SBE reporter transactivation (Fig. 2d). Basal phosphorylation of SMAD3 was increased in ERG-deficient HUVEC compared to control (Supplementary Fig. 3D, F, G). These data indicate that ERG promotes SMAD1 expression and activity, while repressing SMAD2/3 activity through a non-transcriptional mechanism (Fig. 2e).

To investigate whether ERG modulation of SMAD2/3 activity was mediated by the formation of a regulatory complex, we assessed protein interaction by co-Immunoprecipitation (Co-IP) a

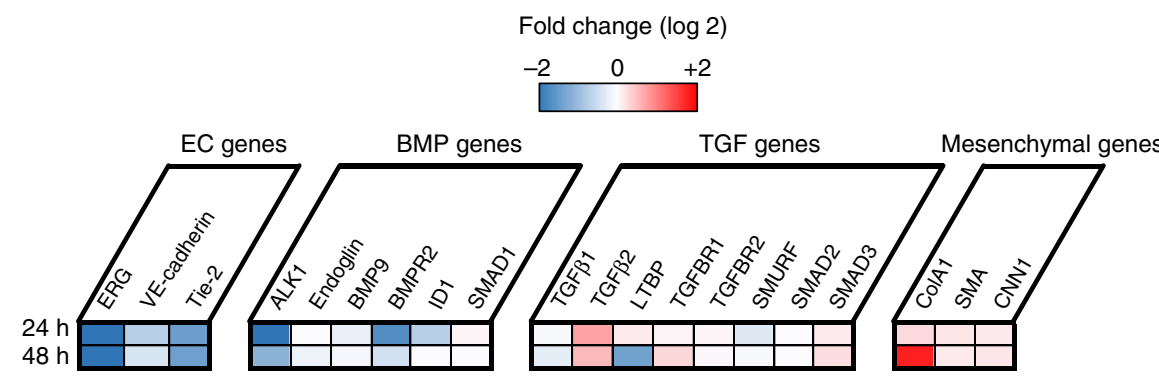

b

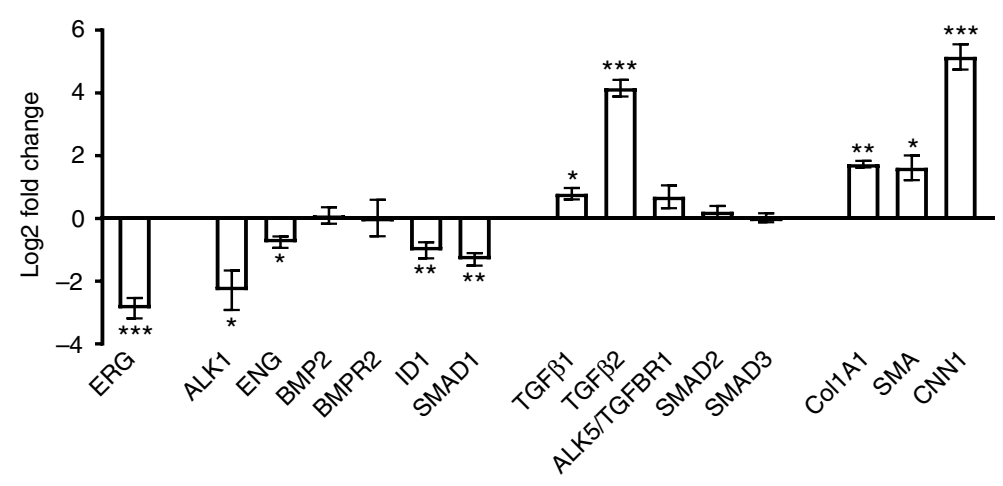

C

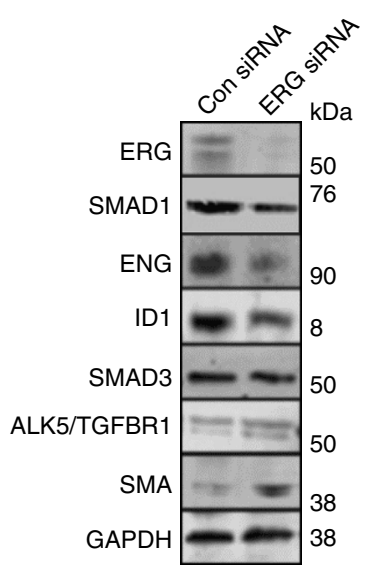

d
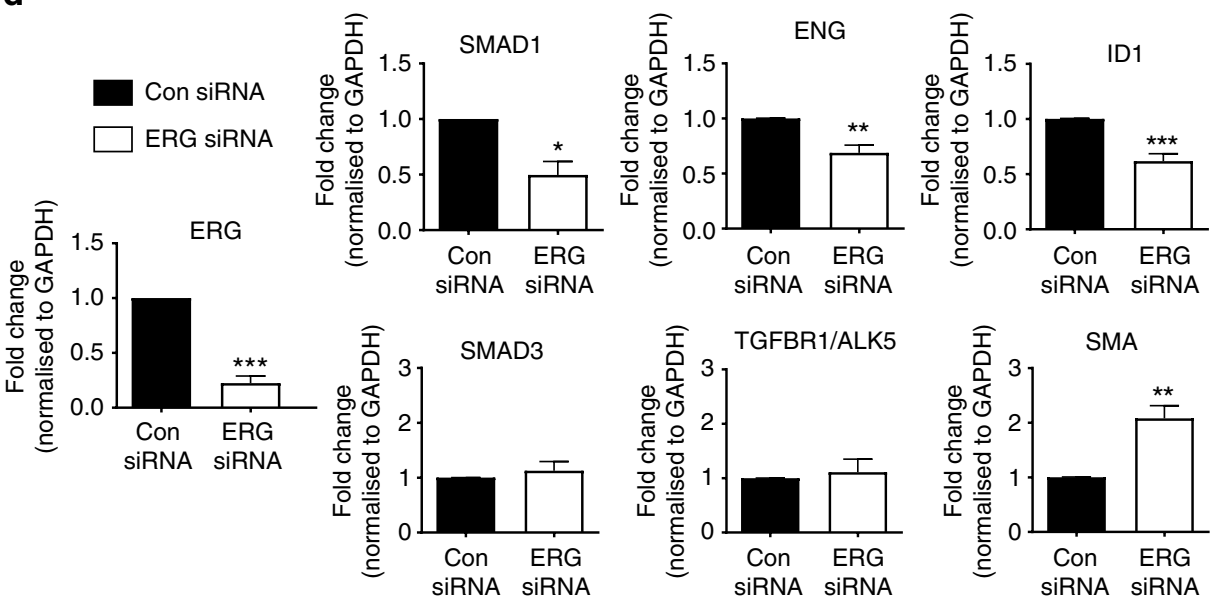

g
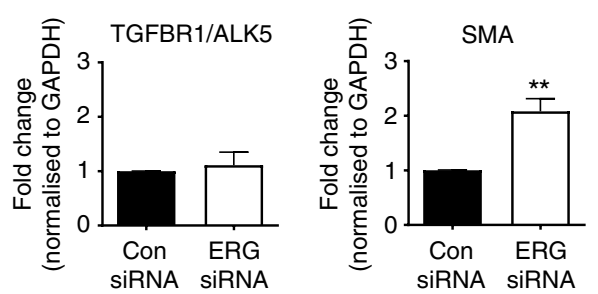

e

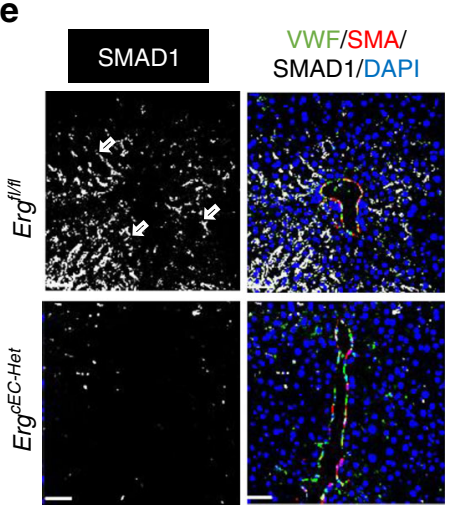

h

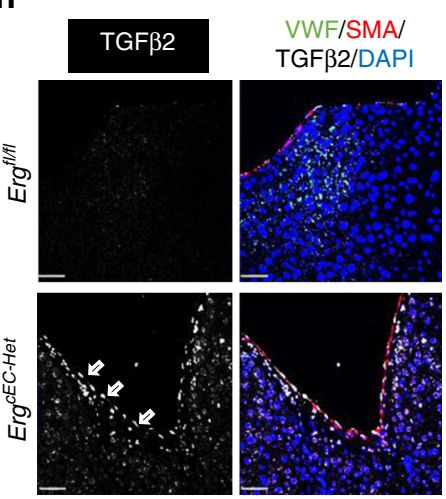


and proximity ligation assay (PLA). Co-IP revealed that ERG interacts with SMAD3 in HUVEC (Fig. 2f, Supplementary Figs. 4A, 5B) and in Hepatic Sinusoidal Endothelial Cells (HSEC; Fig. $2 \mathrm{~g}$ ) isolated from transplant patients with end-stage liver disease. Interestingly, ERG was found to also bind to SMAD2 (Fig. 2f), while no complex formation was observed with SMAD1 in either cell type (Fig. $2 \mathrm{f}$ and Supplementary Fig. 4A). TGF $\beta 2$ treatment resulted in the phosphorylation and translocation of SMAD3 into the nucleus of both HUVEC (Supplementary Fig. 4B) and HSEC (Supplementary Fig. 4C). This was paralleled by increased ERG/pSMAD3 complex formation assessed by CoIP (Fig. 2f; Supplementary Fig. 4A). PLA confirmed that TGF $\beta 2$ treatment induces ERG-pSMAD3 interaction within the nucleus of HUVEC (Fig. 2h) and ERG-SMAD3 in both cell types (Supplementary Fig. 4D); no co-localisation was observed between ERG and SMAD1 (Supplementary Fig. 4D; controls Supplementary Fig. 4E). Inhibition of SMAD2/3 phosphorylation by pretreatment with SB-431542 prevented ERG-SMAD3 complex formation (Fig. 2f), suggesting that this interaction is dependent on SMAD3 phosphorylation. Selective removal of nucleic acids with benzonase treatment from the protein lysates (Supplementary Fig. 5A) did not significantly de-stabilise this interaction (Supplementary Fig. 5B), suggesting that the ERGSMAD3 interaction can be maintained in the absence of DNA. Notably, SB-431542 normalised the enhanced expression of SMAD3-target genes TGF 32 and CNN1 in ERG-deficient HUVEC (Fig. 2i) and HSEC (Fig. 2j), indicating that the EndMT phenotype in ERG-deficient EC is dependent on SMAD2/3 activity. Thus, these data demonstrate that ERG inhibits SMAD2/ 3 activity by direct interaction and formation of an inhibitory complex.

ERG regulates SMAD3-DNA binding to repress SMAD3 activity. Bio-informatic analysis of ERG-repressed-SMAD2/3driven target genes TGF $\beta 2$ and CNN1 revealed the presence of highly conserved ERG DNA binding motifs, upstream of the transcription start site (TSS), which aligned with histone marks for active promoter regions, namely H3K4Me3, H3K27Ac, H3K9Ac and RNA polymerase II (RNA Pol2) occupancy [from Encyclopaedia of DNA Elements (ENCODE)] (Fig. 3a, b). We investigated binding of ERG and SMAD3 to these regions, which also contain several SMAD consensus motifs (Supplementary Fig. 6A, B). Chromatin immunoprecipitation (ChIP)-qPCR showed that in unstimulated HUVEC both ERG (Fig. 3c) and SMAD3 (Fig. 3d) are significantly enriched on the promoters of $T G F \beta 2$ and $C N N 1$. TGF $\beta 2$ stimulation significantly reduced ERG enrichment (Fig. 3c) with concurrent increase in SMAD3 enrichment (Fig. 3d). ERG siRNA treatment led to the loss of ERG enrichment, as expected (Fig. 3e) and resulted in a significant increase in the binding of SMAD3 to the promoters of both target genes (Fig. 3f). These data indicate that ERG inhibits SMAD3 binding to DNA at these target gene promoters (model in Supplementary Fig. 6C).
Erg-deficient mice undergo EndMT and liver fibrogenesis. To determine whether the loss of endothelial ERG expression could influence tissue fibrogenesis in vivo, we assessed tissues from EC-specific constitutive $\mathrm{Erg}$ hemi-deficient mice $\left(\mathrm{Err}^{\mathrm{c}} \mathrm{EC}-\mathrm{Het}\right)$ 23 and EC-specific inducible Pdgfb-iCreER-eGFP/Erg flfl mice $\left(E r g^{i E C-K O}\right.$; genotype data in Supplementary Fig. 7A, B). Increased collagen deposition and elevated SMA expression was most pronounced in the liver of both transgenic lines (Fig. 4a). We observed that reduction or deletion of EC-Erg in both strains caused disrupted portal tracts (schematic Fig. 4b), with significantly increased peri-portal collagen deposition (Fig. 4c) and SMA expression (Fig. 4d; quantification Fig. 4e, f; each genotype was compared to $\left.\mathrm{Erg}^{g l / f l}\right)$. Lineage tracing in $\mathrm{Erg}{ }^{i E C-K O}$ mice revealed $\mathrm{eGFP}^{+} \mathrm{SMA}^{+} \mathrm{EC}$ (Fig. 4g, arrow), a sign of spontaneous EndMT, which was confirmed by quantification of $\mathrm{CD} 31^{+} \mathrm{SMA}^{+}$ double positive cells (representative image Supplementary Fig. 7C, open arrows; quantified in Fig. 4h).

Activation of SMAD3 signalling was observed in the liver endothelium in Erg-deficient lines, corresponding to the in vitro results. pSMAD3 staining, undetectable in littermate control samples, was increased in both $\mathrm{Erg}^{\mathrm{cEC}-h e t}$ and $\mathrm{Erg}{ }^{i E C-K O}$ mice (Fig. 5a, b), in EC as well as surrounding tissue. This was accompanied by proliferation of biliary cells, detected by Ki67 expression, a sign of tissue dysfunction (Supplementary Fig. 7C, D). These data suggest that loss of EC-ERG induces both autocrine and paracrine responses through SMAD3 activation. In parallel with the in vitro studies (Fig. 2i, j), systemic administration of the ALK5 inhibitor SB-431542 abolished spontaneous SMAD3 phosphorylation in $\mathrm{Erg}^{c E C-h e t}$ mice (Supplementary Fig. 8A). Furthermore, in vivo SB-431542 treatment normalised TGFB2 expression in isolated primary mouse EC (Fig. $5 \mathrm{c}$ ) and normalised both SMA expression (Fig. 5d, e) and collagen deposition (Fig. 5f and Supplementary Fig. 8B) compared with vehicle (DMSO)-treatment in $\mathrm{Erg}{ }^{i E C-K O}$ mice. These data show that loss of endothelial ERG expression causes enhanced SMAD3 activity in both EC and surrounding tissue, resulting in spontaneous EndMT and a pro-fibrotic microenvironment within the liver.

Loss of ERG in murine and human fibrotic liver disease. The finding of spontaneous EndMT and fibrogenesis in Erg-deficient mice led us to investigate whether endothelial ERG plays a role in the pathophysiology of liver fibrosis, using a carbon tetrachloride $\left(\mathrm{CCL}_{4}\right)$-induced mouse model. Chronic administration of $\mathrm{CCL}_{4}$ for 8 weeks resulted in characteristic bridging fibrosis (Supplementary Fig. 9A), increased SMA (Fig. 6a, b) and pSMAD3 expression (Supplementary Fig. 9B) in peri-portal areas. Chronic $\mathrm{CCL}_{4}$ treatment caused loss of ERG expression in all EC, which was partially restored following an additional 3-week recovery period (Fig. 6a, c). ERG expression is downregulated by inflammatory stimuli such as TNF- $\alpha^{26}$ and LPS ${ }^{27}$. As elevated inflammatory cytokines are associated with fibrogenesis, we assessed the impact of TNF- $\alpha$ inhibition on ERG expression (Fig. 6d) and

Fig. 1 Differentially expression of canonical TGF $\beta / B M P-S M A D$ genes in ERG-deficient HUVEC. a Microarray analysis of ERG-dependent genes in HUVEC was performed at 24 and $48 \mathrm{~h}$ after ERG depletion, as described ( $n=3$ biological replicates) ${ }^{24}$; fold change (log 2 ) of selected TGF $\beta /$ BMP associated genes represented as high (red) and low (blue) expression compared to the median (white). Gene expression data were validated in HUVEC transfected with Control (Con siRNA) or ERG siRNA by $\mathbf{b}$ quantitative PCR and $\mathbf{c}$ immunoblotting, showing reduction of ERG protein levels following siRNA, $\mathbf{d}$ quantification by fluorescence intensity normalised to GAPDH for ERG, SMAD1, ENG, ID1, SMAD3, ALK5 and SMA ( $n=5$ ). e Representative images of SMAD1 expression (white; arrows identify expression in the sinusoidal endothelium), VWF (green), SMA (red) and DAPI (blue) in liver tissue from Erg fl/fl and $\mathrm{Erg}^{\mathrm{cEC} \text {-het }}$ mice (Scale bar $50 \mu \mathrm{m}$ ). $\mathbf{f}$ Quantitative analysis of TGF $\beta 2$ protein expression was performed by ELISA in whole cell HUVEC lysates $(n=7)$. g Representative image of TGF $\beta 2$ expression in HUVEC transfected with control or ERG siRNA by immunofluorescence; nuclei are identified by DRAQ $\mathrm{V}$ and cells are co-stained for ERG (green). Scale bar $20 \mu \mathrm{m}$. h Representative images of TGF $\beta 2$ expression (white; arrows) in large vessel within the liver

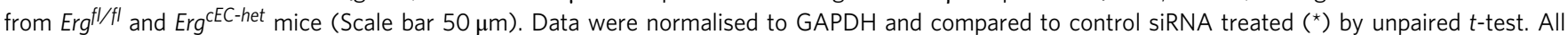
graphical data are mean \pm s.e.m., ${ }^{\star} P<0.05,{ }^{\star \star} P<0.01,{ }^{\star \star \star} P<0.001$ 
SMAD3 activity (Fig. 6e) by co-administration of the TNF- $\alpha$ antagonist etanercept in an acute $\mathrm{CCL}_{4}$-induced liver injury. A single $\mathrm{CCL}_{4}$ injection caused a significant increase in SMA (Fig. 6f), reduction of ERG expression (Fig. 6g) and enhanced phosphorylation of SMAD3 at $48 \mathrm{~h}$ (Fig. $6 \mathrm{~h}$ and Supplementary Fig. 9B); co-administration of etanercept significantly reduced $\mathrm{CCL}_{4}$-induced SMA expression (Fig. 6f), normalised endothelial ERG expression (Fig. 6g) and reduced phosphorylation of SMAD3 (Fig. 6h and Supplementary Fig. 9C). However, etanercept was unable to correct the CCL4-induced injury in $\mathrm{Erg}^{\mathrm{CEC}-h e t}$ mice (Fig. 6i), with no normalisation of transcriptional target genes (Fig. 6j) or SMA expression in the liver (Fig. 6k). a

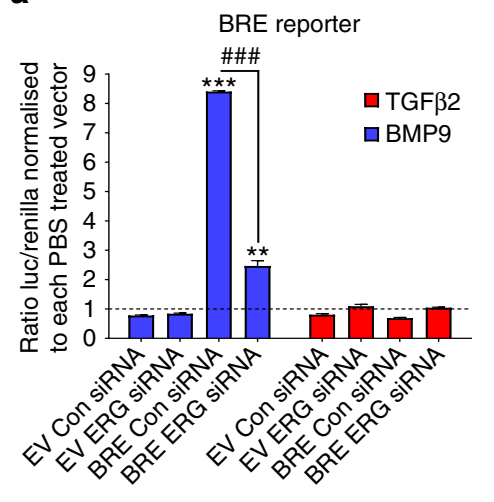

C

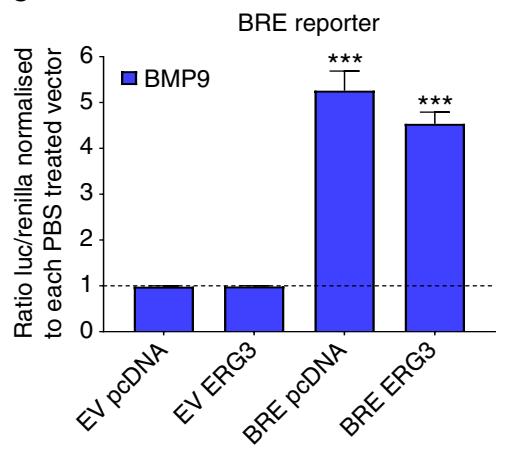

f

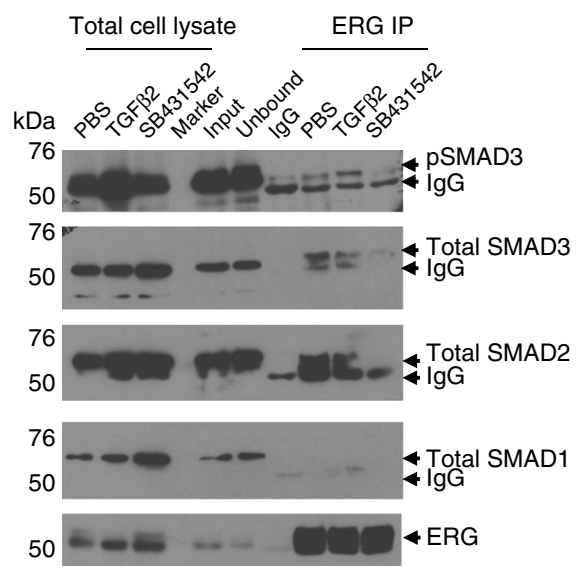

i

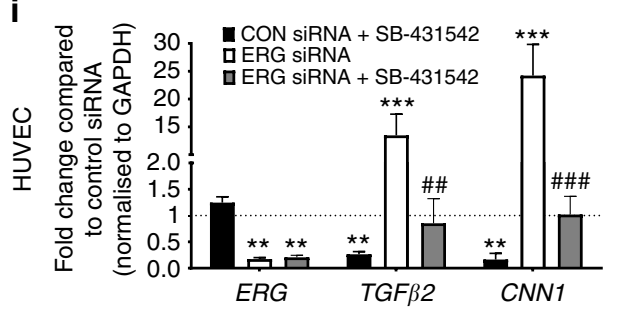

b

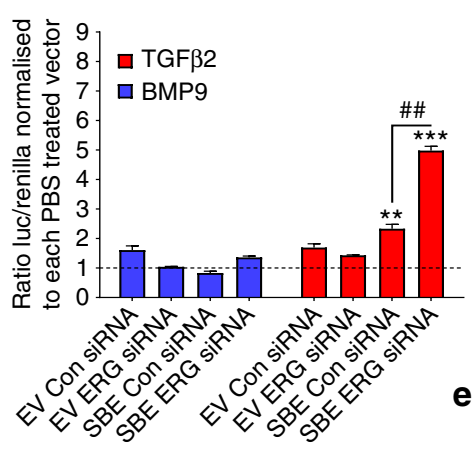

d

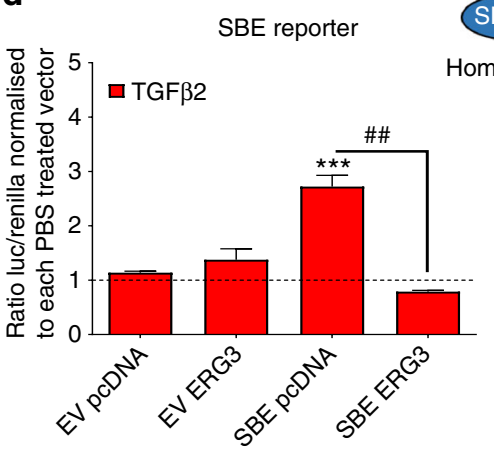

g

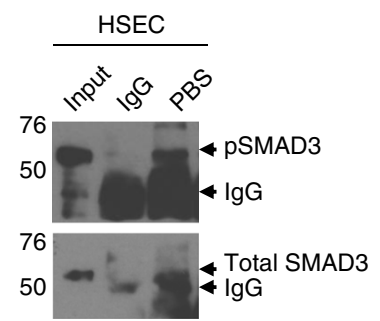

h

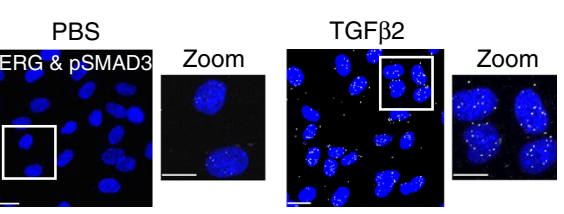

j

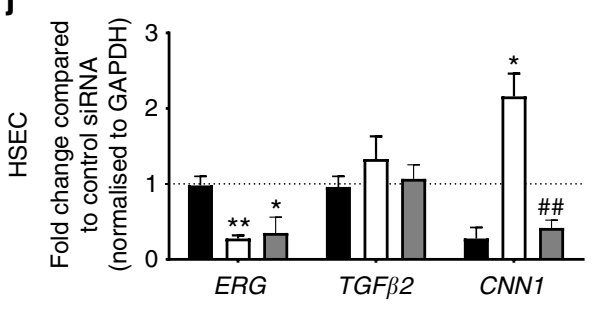


These data suggest that the TNF- $\alpha$ antagonist protects from $\mathrm{CCL}_{4}$-induced tissue injury through the endothelial ERG pathway, and reveal that ERG expression is an early, sensitive marker of vascular inflammation during liver fibrogenesis, which can be modulated by clinically relevant therapies.

Finally, to verify the relevance of this pathway in human diseases associated with fibrosis, we obtained liver tissue samples from end-stage liver transplant patients with alcoholic liver disease (ALD), non-alcoholic steato-hepatitis (NASH) or primary biliary cirrhosis (PBC); non-fibrotic tissue from separate donors was used as control. Liver sections were profiled by immunofluorescence microscopy for CD31, SMA and ERG expression (Fig. 7a). In non-fibrotic human liver samples, ERG was expressed in both vascular and sinusoidal EC, with characteristic nuclear localisation (Supplementary Fig. 10A, B). ERG expression was significantly decreased in $\mathrm{CD} 31^{+}$EC from ALD and PBC patient samples compared to non-fibrotic liver samples; in samples from NASH patient the decrease in EC-ERG expression did not reach significance (Fig. $7 \mathrm{~b}$; additional images provided in Supplementary Fig. 11A-D). EndMT, identified by CD $31^{+} \mathrm{SMA}^{+}$ double positive cells, was identified in liver fibrosis associated with ALD and PBC but not in NASH patients (Fig. 7c). Analysis of $\mathrm{CD}_{31}{ }^{+} \mathrm{SMA}^{+}$co-localisation with ERG expression in all patient samples revealed a significant negative correlation between ERG expression and EndMT (Fig. 7d). Therefore, loss of ERG expression appears to be a sensitive marker of inflammatorydriven fibrogenesis and could represent a novel biomarker for EndMT in human fibrotic liver.

\section{Discussion}

In this study, we report that the endothelial transcription factor ERG controls the homoeostatic balance of TGF $\beta / B M P$ canonical signalling in vitro and in vivo; loss of endothelial ERG results in EndMT and spontaneous liver fibrosis in mouse models and inversely correlates with EndMT and liver fibrosis in patients.

Expression profiling of ERG-deficient HUVEC identified dysregulation of genes involved in SMAD1 and SMAD2/3 signalling. Expression of EC-restricted genes SMAD1 and its co-receptors $A C V R L 1$ and ENG was reduced in ERG-deficient HUVEC, in line with the previous report of ENG as a direct ERG target $^{33}$. Mutations in ACVRL1 and ENG cause hereditary haemorrhagic telangiectasia (HHT), a disease characterised by vascular malformations 34,35 , in agreement with the model that this arm of the BMP/TGF $\beta$ pathway is required for vascular homoeostasis. Our data reveal that loss of ERG expression significantly affects SMAD1 phosphorylation and transcriptional activity. Co-IP and PLA assays revealed that ERG does not interact with SMAD1, suggesting that ERG is primarily required for the expression of key proteins in the SMAD1 pathway.
In ERG-deficient EC, loss of SMAD1-dependent transcription was accompanied by enhanced expression of SMAD2/3 target genes, indicating an inverse relationship between ERG and SMAD2/3-dependent targets. This shift in SMAD signalling is consistent with an EndMT phenotype, characterised by the loss of EC identity and the emergence of a mesenchymal gene expression profile ${ }^{9}, 10,13$. We demonstrate that ERG directly represses SMAD2/3 transcriptional activity, independently of expression of SMAD2/3 or its receptor ALK5. We find that ERG can interact with SMAD2/3 in HUVEC and in HSEC in agreement with studies that show overexpression of exogenous ERG and SMAD3 results in protein interaction ${ }^{36,37}$. ERG and SMAD3 both bind to the promoters of target genes, indicating that ERG acts as a co-repressor for SMAD3. These data are in line with a study mapping SMAD2/3 global DNA binding in keratinocytes, which revealed that ETS motifs are represented in $\sim 50 \%$ of SMADenriched genes ${ }^{38}$, suggesting that ETS/SMAD dual motifs may be important in the regulation of TGF $\beta / S M A D$ signalling.

Previous work investigating the regulation of TGF $\beta / \mathrm{BMP}$ signalling by ERG in vivo has focused on developmental biology. ERG was shown to be involved in endocardial-mesenchymal transition in cardiac valve morphogenesis ${ }^{39}$ and for TGF $\beta$ mediated differentiation of sclerotome cells during vertebrae bone development ${ }^{36}$. Here we utilised two EC-specific Erg-deficient mouse strains to assess the role of ERG in regulating SMAD signalling in post-natal liver physiology; this is the first study to identify ERG expression in liver vascular and sinusoidal EC. Both strains displayed deformation of portal tracts with increased collagen deposition, SMA and PSMAD3 expression confirming a pro-fibrotic phenotype. Furthermore, analysis of $\mathrm{CD}_{3}{ }^{+} \mathrm{SMA}^{+}$ double positive EC and lineage tracing indicates that Erg-deficient EC undergo spontaneous EndMT in vivo, which was dependent on SMAD3, as shown by rescue of the phenotypes with SB-431542. Interestingly, endothelial ERG deficiency caused enhanced pSMAD3 not only in the endothelium, but also in the surrounding tissue. Thus, these data suggest that ERG is central to EC-specific regulation of SMAD2/3 and promotes tissue homoeostasis by preventing a paracrine TGF $\beta 2$-SMAD3 positive feedback loop, resulting in a pro-fibrotic tissue microenvironment.

In a murine model of acute and chronic $\mathrm{CCL}_{4}$-induced liver fibrosis, we observed a rapid, marked and persistent reduction in ERG expression. Notably, ERG expression was partially restored in recovering tissue ( 3 weeks after terminating chronic $\mathrm{CCL}_{4}$ administration) and protected by co-administration of the TNF- $\alpha$ inhibitor etanercept. By profiling ERG expression in human liver, we found marked loss of ERG expression in end-stage liver tissues from ALD and PBC patients. These diseases have been associated with inflammatory pathways ${ }^{40,41}$. Loss of ERG expression could be due to chronic vascular inflammation; a similar observation

\footnotetext{
Fig. 2 ERG differentially regulates transcriptional activity of SMAD1 and SMAD3. ERG-dependent regulation of SMAD1 and SMAD3 activity were assessed by transactivation assays using either SMAD1 reporter pGL3-BRE (BRE reporter, $\mathbf{a}$ and $\mathbf{c}$ ) or SMAD3 reporter pBV-SBE4 (SBE reporter, b and $\mathbf{d}$ ). HUVEC were either co-transfected with Control siRNA (Con siRNA) or ERG siRNA for $24 \mathrm{~h}$ (a and $\mathbf{b}$ ) or with pcDNA or ERG3 overexpression construct (ERG3) (c and d) prior to treatment with TGF $\beta 2$ (red) and BMP9 (blue) or PBS (dashed line) for $18 \mathrm{~h}$ (data from pooled HUVEC in triplicate experiments). The ratio of luciferase to renilla from each transfection was normalised to PBS-treated control or to groups co-transfected with (a and $\mathbf{b}$ ) Con siRNA or (c and $\mathbf{d}$ ) pcDNA $(n=3)$. e Schematic of the inverse regulation of SMAD1 and SMAD3 by ERG. $\mathbf{f}$ Protein-protein interactions between ERG, SMAD1, SMAD2 and SMAD3 were assessed by Co-IP assay in whole cell lysate from HUVEC. SB-431542 (SB; $10 \mu \mathrm{M})$ treatment was performed for $1 \mathrm{~h}$ and TGF $\beta 2\left(10 \mathrm{ng} \mathrm{ml}^{-1}\right.$ ) or PBS treatments were performed 30 min prior to lysis. Lysates were immunoprecipitated with mouse lgG or mouse $\alpha$-ERG and then immuno-blotted for $\alpha$-ERG, $\alpha$-SMAD1, $\alpha$-SMAD2, $\alpha$-SMAD3 and $\alpha$-pSMAD3 (Ser423/425). Images representative of four experiments. $\mathbf{g}$ ERG-SMAD3 interaction was assessed by Co-IP in untreated HSEC. $\mathbf{h}$ Cellular localisation of the interaction between ERG with pSMAD3 was investigated by Proximity Ligation Assay (PLA) following 30 min TGF $\beta 2$ treatment in HUVEC $(n=2)$. i HUVEC or $\mathbf{j}$ HSEC were pre-treated with SB-431542 or DMSO prior to transfection with either control siRNA or ERG siRNA for $48 \mathrm{~h}$ and analysed by qPCR $(n=3)$. Data were normalised to GAPDH and compared to control siRNA treated ( $\left.{ }^{\star}\right)$ or to ERG siRNA treated with DMSO (\#) by unpaired $t$-test. All graphical data are mean \pm s.e.m., ${ }^{\star}$ or ${ }^{\#} P<0.05,{ }^{\star \star}$ or ${ }^{\# \#} P<0.01$, ${ }^{\star \star \star}$ or ${ }^{\# \# \# P<0.001}$
} 
a

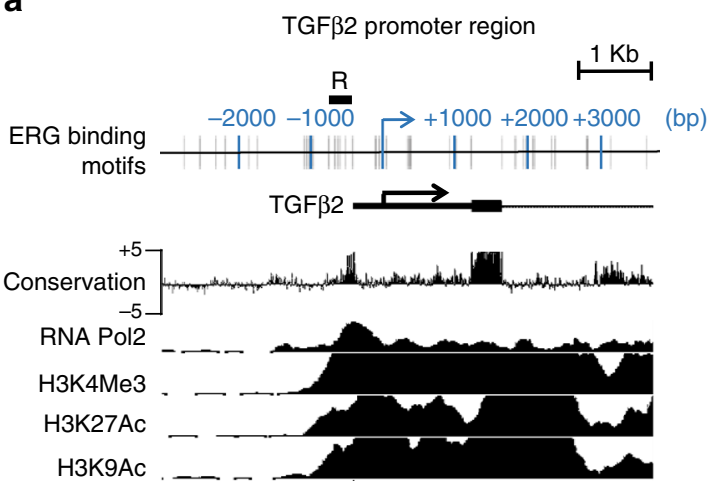

b

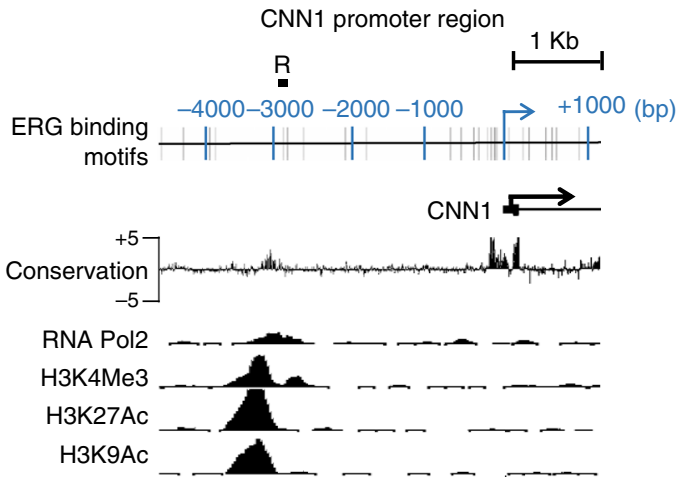

$\mathrm{R}=$ Primer spanning promoter region

C

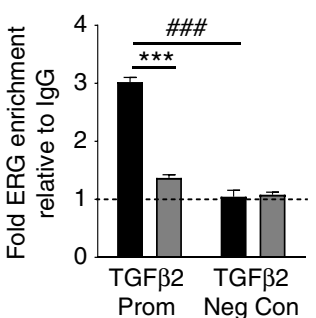

ERG ChIP

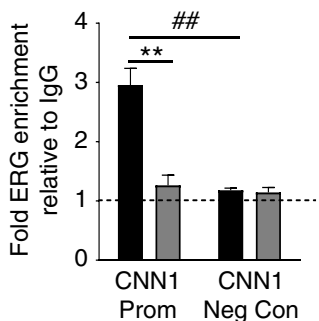

e

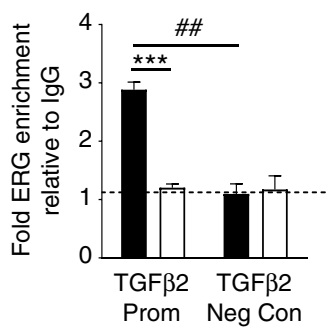

ERG ChIP

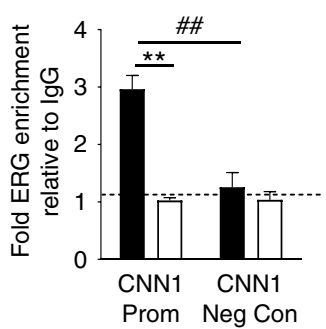

d

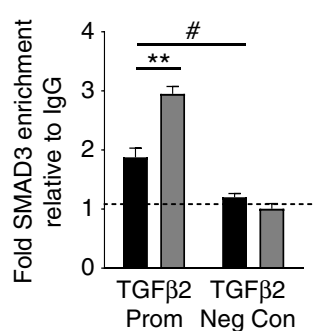

SMAD3 ChIP

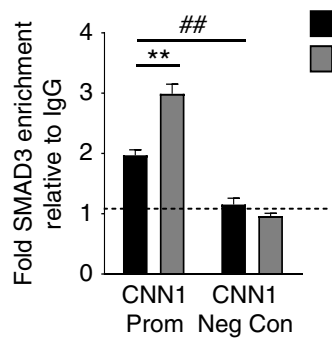

f

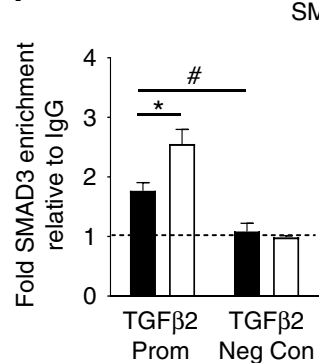

PBS

TGF $\beta 2$

Fig. 3 ERG is a key factor for endothelial specific inhibition of SMAD3-DNA binding. Putative ERG binding sites (grey bars) are located within the a TG $\beta 2$ and $\mathbf{b}$ CNN1 promoters upstream of the transcription start site (TSS) (arrow); ENCODE sequence conservation between 100 vertebrates across this region is shown. ENCODE ChIP-seq data profile for H3K4Me3, H3K27Ac, H3K9Ac and RNA polymerase II (RNA Pol2) in HUVEC indicate open chromatin and active transcription. Location of qPCR amplicon region $\mathrm{R}$ is indicated. ChIP-qPCR analysis of HUVEC was assessed following treatment with $\mathbf{c}$ and $\mathbf{d}$ TGF $\beta 2$ or PBS for $30 \mathrm{~min}$, or $\mathbf{e}$ and $\mathbf{f}$ control or ERG siRNA. Chromatin was immunoprecipitated with $\mathbf{c}$ and $\mathbf{e}$ an $\alpha$-ERG antibody, $\mathbf{d}$ and $\mathbf{f} \alpha$-SMAD3 antibody or control IgG. DNA was analysed by qPCR comparing specific primers against a negative control region for each target. Results are expressed as fold change compared to IgG ( $n=3$ for all experiments). Basal enrichment was analysed compared to negative control region (\#). TGF $\beta 2$ or ERG siRNA treatment were compared to PBS treated or control siRNA $\left(^{\star}\right)$ mean \pm s.e.m., ${ }^{\star}$ or ${ }^{\#} P<0.05$, ${ }^{\star \star}$ or ${ }^{\# \#} P<0.01$, ${ }^{\star \star \star}$ or ${ }^{\# \# \#} P<0.001$ by unpaired $t$-test

was reported in the endothelium overlaying human atherosclerotic plaques ${ }^{26}$. Anti-TNF therapy has been effective in preclinical liver fibrosis models ${ }^{42}$, 43 ; furthermore, etanercept has shown some efficacy in a case study of PBC associated with rheumatoid arthritis ${ }^{44}$. Since ERG expression can be downregulated by inflammatory agents, it is likely that inflammation plays a key role in the loss of ERG expression in these patients. Interestingly, etanercept could restore ERG expression and reduce SMAD3 activity in the in vivo model of $\mathrm{CCL}_{4}$-induced liver fibrosis in wild-type mice, but was ineffective in $\mathrm{Erg}^{\mathrm{cEC}-h e t}$ mice, suggesting that anti-TNF therapies exert a beneficial effect by restoring endothelial homoeostasis through the ERG pathway.

In conclusion, our findings identify ERG as a novel EC-specific regulator of canonical TGF $\beta / \mathrm{BMP}$, promoting EC-dependent tissue homoeostasis. We provide evidence that loss of ERG expression is an early, causative event during liver fibrogenesis. We describe EndMT in human liver disease, which showed pathological differences between ALD, PBC and NASH patients. Therefore, we propose that ERG may represent a sensitive tissue biomarker to monitor vascular dysfunction and influence therapeutic strategies.

\section{Methods}

Cell culture. Primary HUVEC were collected from umbilical cords using $0.5 \mathrm{mg} \mathrm{ml}^{-1}$ Collagenase dissolved in HBSS warmed to $37^{\circ} \mathrm{C}$. Isolated cells were cultured in M199 media supplemented with Endothelial cell growth supplement (Sigma) and $20 \%$ bovine calf serum ${ }^{22}$. Hepatic sinusoidal endothelial cells (HSEC) were isolated from $\sim 30 \mathrm{~g}$ human liver tissue obtained from explanted livers collected from patients in the Liver Unit at Queen Elizabeth Hospital in Birmingham with informed consent and ethics committee approval. HSEC were then cultured in medium composed of human endothelial basal growth medium (Invitrogen), 10\% $\mathrm{AB}$ human serum (HD Supplies), $10 \mathrm{ng} \mathrm{ml}^{-1}$ vascular endothelial growth factor (VEGF), and $10 \mathrm{ng} \mathrm{ml}^{-1}$ hepatocyte growth factor (HGF) (PeproTech). Human ERG expression was repressed by transfection of cells with $20 \mathrm{nM}$ siRNA against ERG exon 6 (Qiagen; 5'-CAGATCCTACGCTATGGAGTA-3') or a second siRNA (\#2) targeting exon 7 (Invitrogen; 5'-ACTCTCCACGGTTAATGCATGCTAG-3') 
a
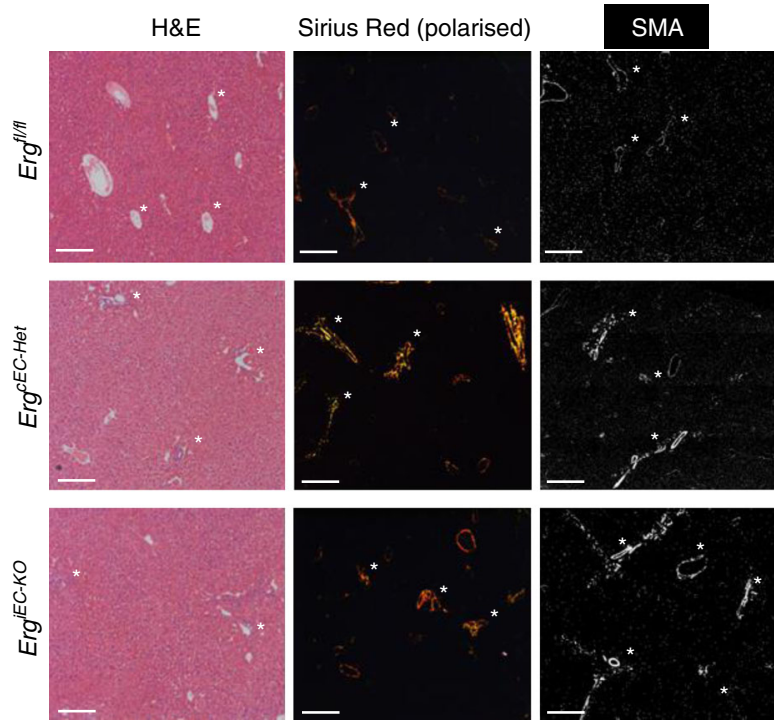

d
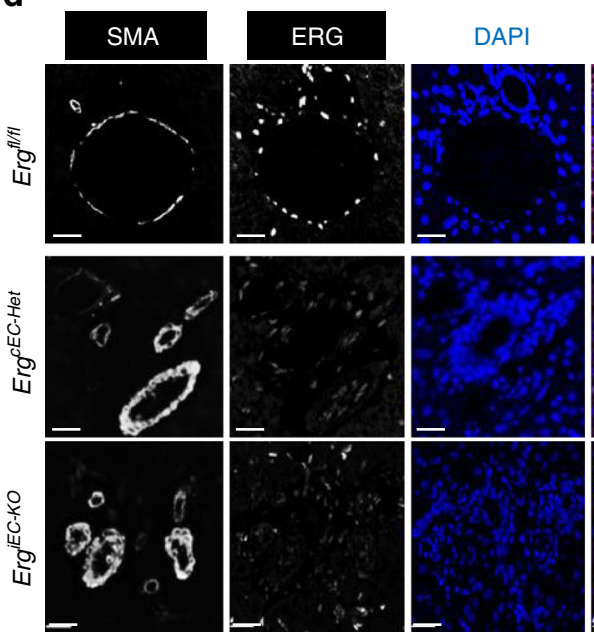

g
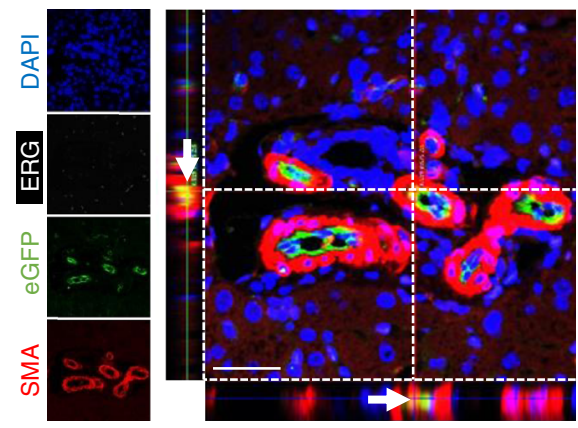

b

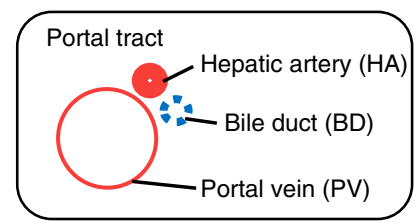

C

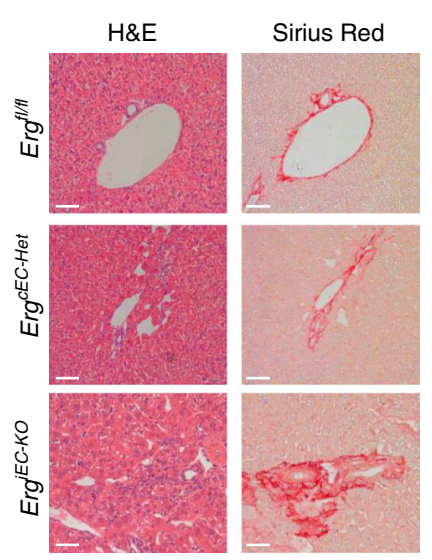

e SMA/ERG/DAPI
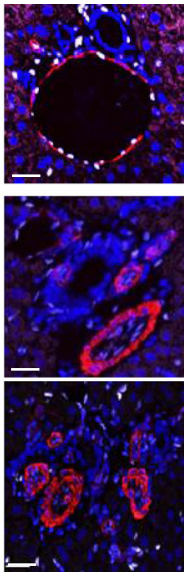

$\mathbf{h}$

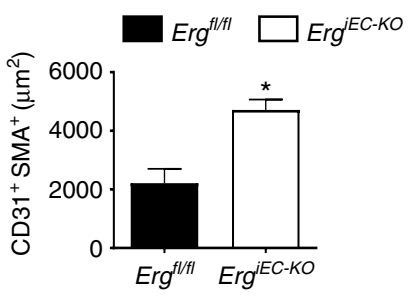

Fig. 4 ERG-deficient mouse displays spontaneous liver fibrogenesis surrounding portal tracts. a EC-specific constitutive Erg hemi-deficient (Erg ${ }^{c E C-h e t}$ ) and inducible PDGFB-eGFP-Cre ERG flox homozygous ( $\mathrm{Frg}^{\mathrm{iEC}-\mathrm{KO}}$ ) mice aged between 8 and 10 weeks were imaged by H\&E, picro-sirius red and SMA (Scale bar $500 \mu \mathrm{m})$. Portal tract regions are identified by white asterisk with components depicted schematically in $\mathbf{b}$. Portal tracts were assessed by $\mathbf{c} \mathrm{H} \& \mathrm{E}$ and picrosirius (red) (Scale bar $20 \mu \mathrm{m}$ ). d Immunofluorescence for SMA (grey scale; red in merge), ERG (white), DAPI (blue) and merged (Scale bar $20 \mu \mathrm{m}$ ). Images captured from e Erg ${ }^{C E C-h e t}$ and $\mathbf{f} E r g^{i E C-K O}$ mice were quantified for areas of Picro-sirius red positive tissue, using polarised light, and SMA expression ( 3 fields per mouse, $n=3$ ). Scale bar $50 \mu \mathrm{m}$. $\mathbf{g}$ Co-localisation of SMA and eGFP expression, indicative of EndMT, was observed in Erg ${ }^{i E C-K O}$ mice by immunofluorescence (double positive cell indicated by arrow in cross-section). $\mathbf{h}$ Quantification of $\mathrm{CD}_{31}{ }^{+} \mathrm{SMA} \mathrm{A}^{+}$double positive cells (three fields per mouse, $n=3-4)$. Data were compared to $\mathrm{Erg}^{f l / f l}$ littermate controls $\left(^{\star}\right)$ by unpaired $t$-test. All graphical data are mean \pm s.e.m., ${ }^{\star} P<0.05,{ }^{\star \star} P<0.01$ 
a

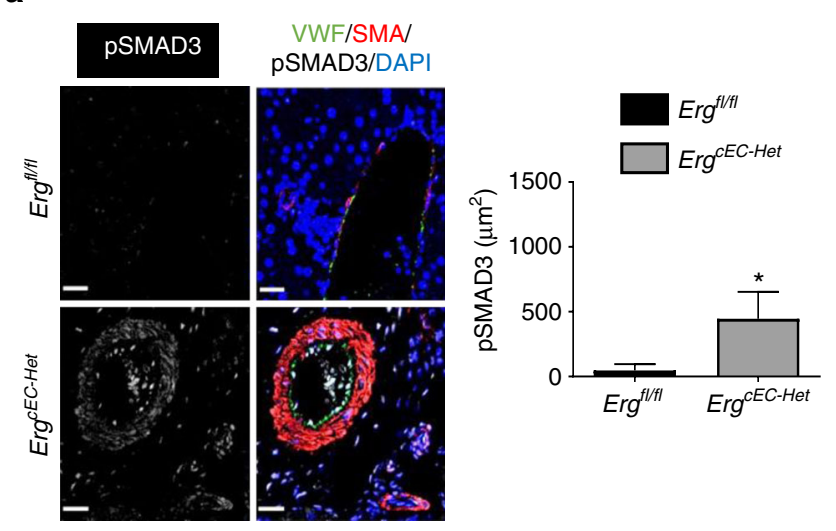

b

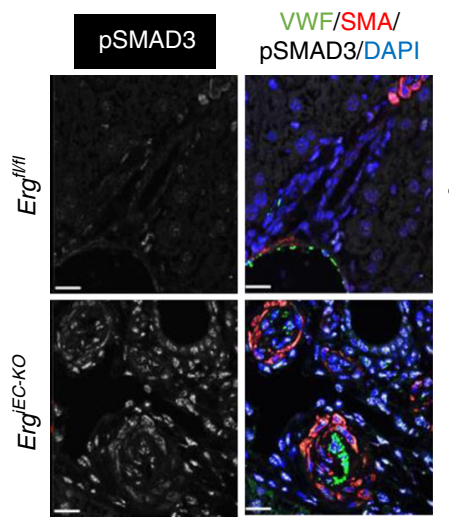

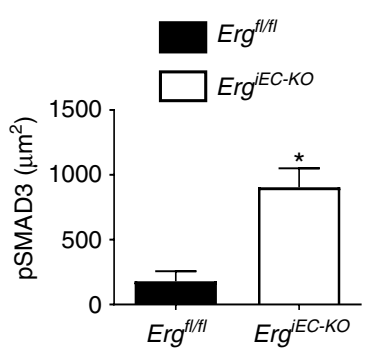

C

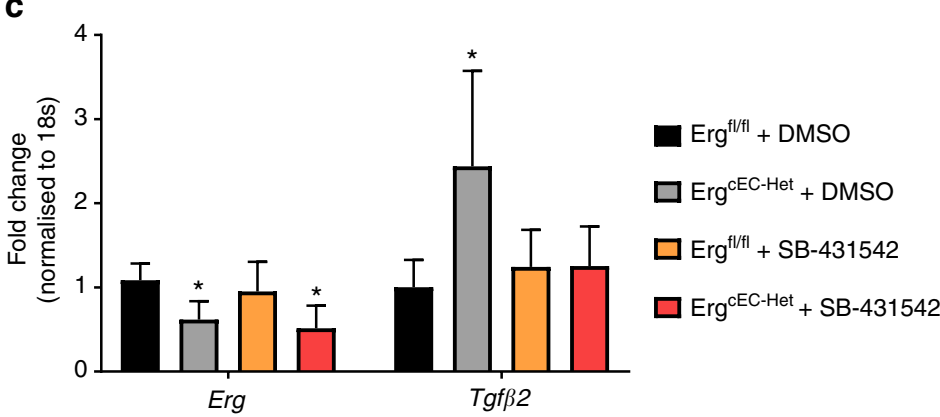

d

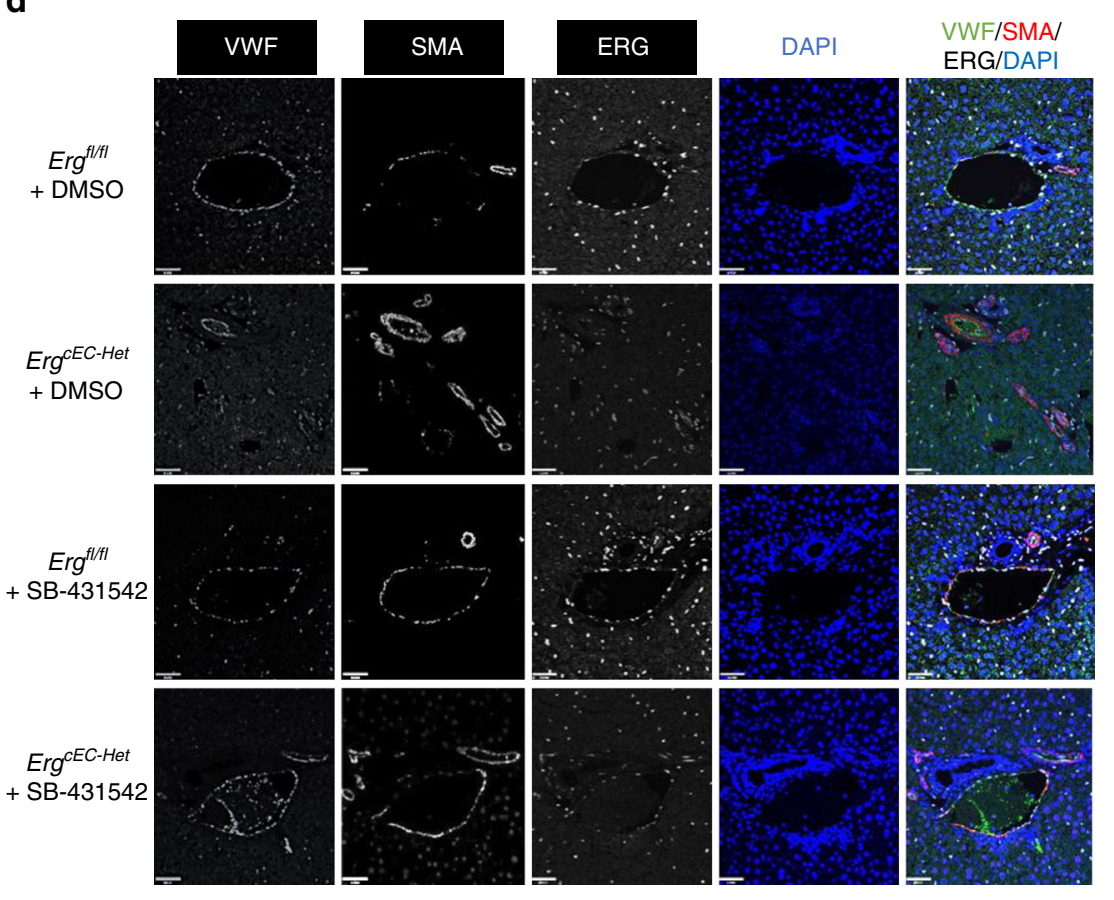

e
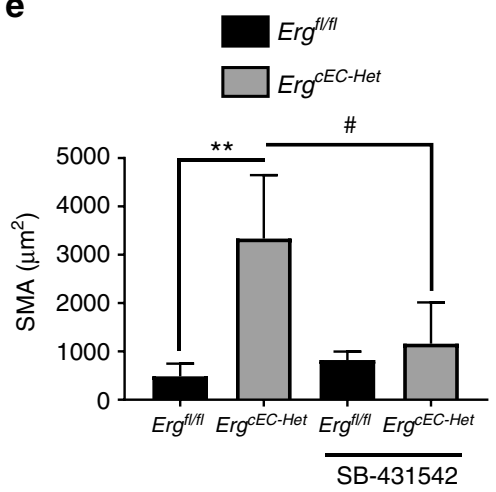

f

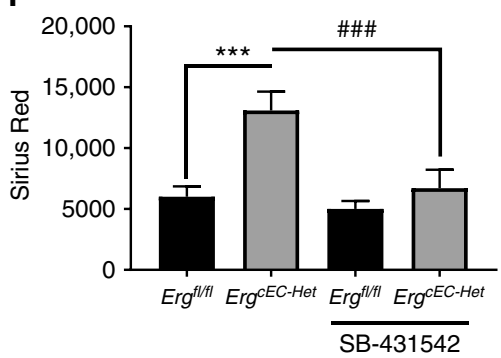

Fig. 5 Peri-portal ERG-deficient mouse fibrogenesis phenotype is SMAD3-dependent. a, b SMAD3 activity in portal tract regions was assess by

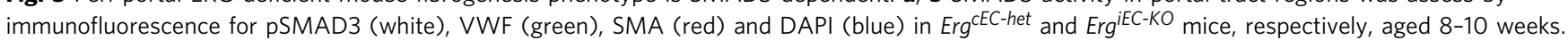
Quantification of pSMAD3 area (three fields per mouse, $n=3)$. SB-431542 (10 $\mathrm{mg} \mathrm{kg}^{-1}$, i.p) was administered in Erg ${ }^{c E C-h e t}$ mice three times a week for 2 weeks. Scale bar $20 \mu \mathrm{m}$. c mRNA was isolated from CD31+ murine EC isolated from lung tissue and analysed by qPCR $(n=4)$. Data were compared to EC from Erg ${ }^{f l / f l}$ littermate controls $\left(^{\star}\right)$ or to DMSO treated Erg ${ }^{c E C-h e t}(\#)$ by one-way ANOVA and Bonferroni multiple comparison post-test. d Representative images of portal tract VWF (grey scale; green in merge), SMA (grey scale; red in merge), ERG (white), DAPI (blue) and merged panels (Scale bar $50 \mu$ m). e Quantification of SMA expression ( 5 fields per mouse, $n=4$ ). $\mathbf{f}$ Quantitative analysis of Picro-sirius red positive tissue, using polarised light (more representative images in Supplementary Fig. 8B). Data were compared to Erg ${ }^{f l / f l}$ littermate controls $\left(^{\star}\right)$ or to DMSO treated ErgcEC-het (\#) by one-way ANOVA and Bonferroni multiple comparison post-test. All graphical data are mean \pm s.e.m., ${ }^{\star}$ or ${ }^{\#} P<0.05,{ }^{\star \star}$ or ${ }^{\# \#} P<0.01,{ }^{\star \star \star}$ or ${ }^{\# \# \# P<0.001}$ 
of the ERG locus, both are denoted in the text as ERG siRNA. In parallel, an AllStars Negative Control siRNA (Qiagen) was used.

Pharmacological growth factor in vitro cell treatments. SB- 431542 was purchased from Sigma (UK). SMAD3-dependent SMAD-Binding Element (SBE) reporter (SBE4-Luc) was a gift from Bert Vogelstein (Addgene plasmid \#16495) ${ }^{30}$ pBV-Luc empty vector, lacking a promoter sequence, was a gift from Bert

Vogelstein (Addgene plasmid \#16539) and was used as a control ${ }^{45}$. BMP response element (BRE) luciferase reporter (pGL3-BRE Luciferase) was a gift from Martine Roussel and Peter ten Dijke (Addgene plasmid \#45126) ${ }^{29}$. Human ERG cDNA a

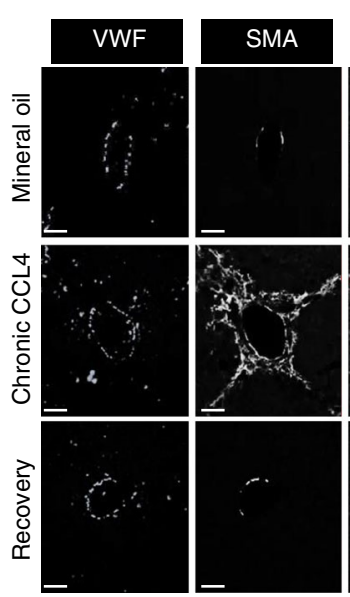

d

Mineral oil

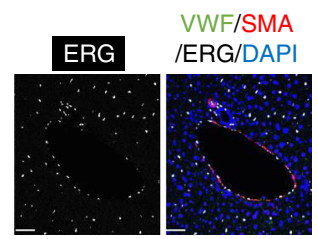

f

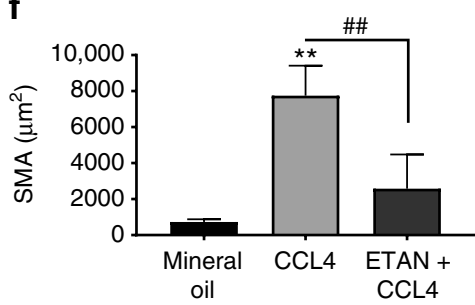

i

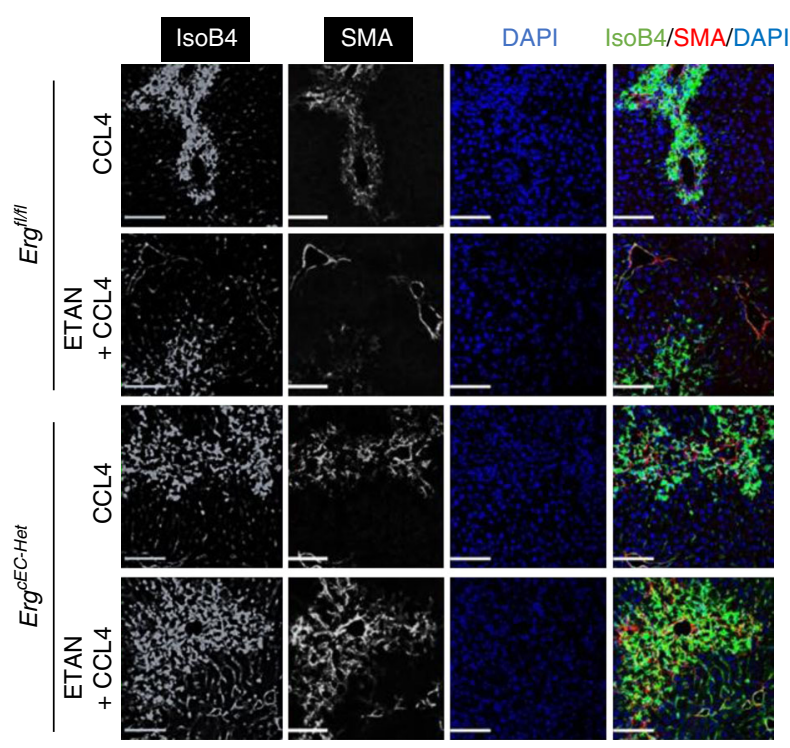

g b

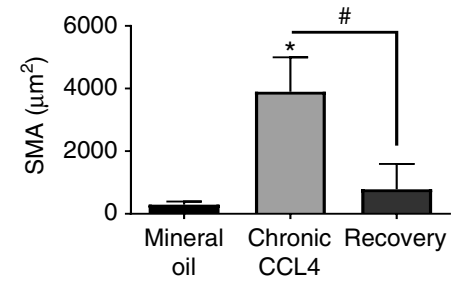

C

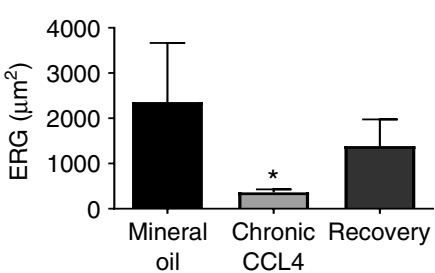

e

Etanercept +48 h CCL4

VWF/SMA/ $48 \mathrm{~h} C \mathrm{Cl} 4 \mathrm{Etanercept}$

VWF/SMA/ PSMAD3/DAPI
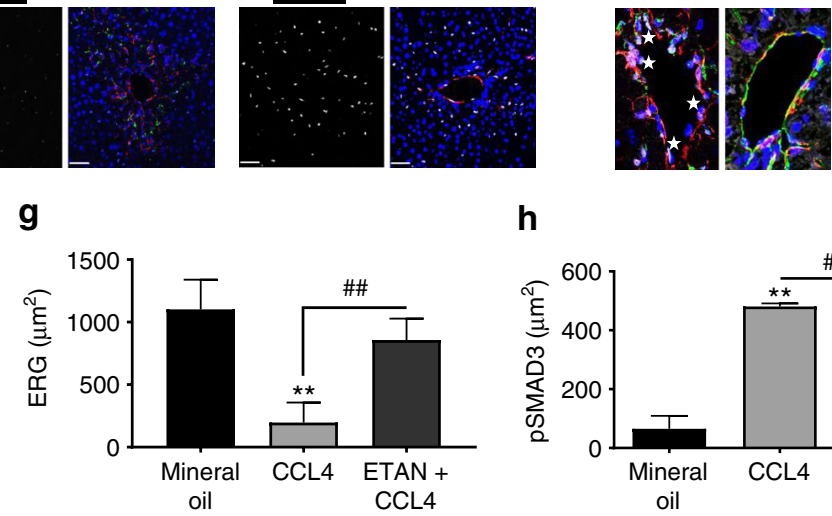

h

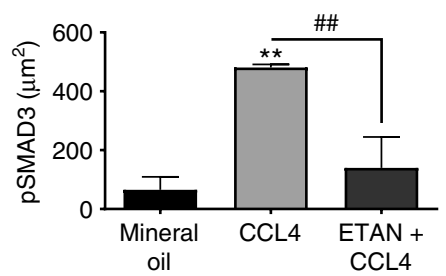

j

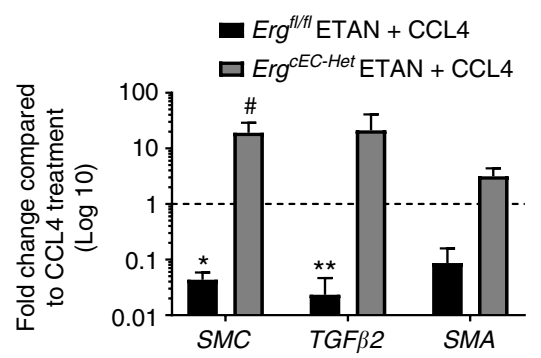

k

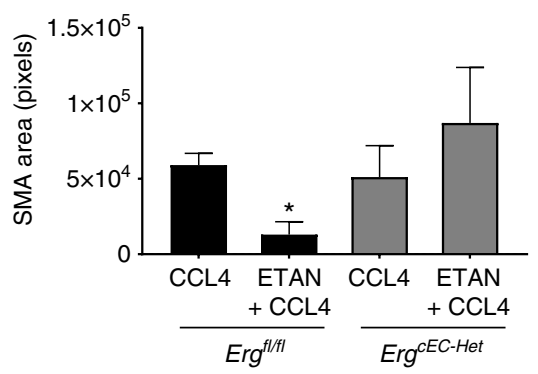


(NCBI Accession NM_182918) was cloned into the mammalian expression vector pcDNA3.1 (Invitrogen). pGL4.10[luc2] (Promega, Madison, USA) Firefly Luciferase empty vector, lacking a promoter sequence, was used as a control. pGL4.73 [hRluc/SV40] (Promega) Renilla luciferase vector was used as an internal normalisation control in the luciferase assay. HUVEC were transfected with Genejuice transfection reagent (Merck Chemicals), as recommended. Cells were incubated with $9 \mu \mathrm{l}$ of GeneJuice, $1 \mu \mathrm{g}$ luciferase plasmid and/or $1 \mu \mathrm{g}$ of expression plasmid and $1 \mu \mathrm{g}$ of pGL4-Renilla for $24 \mathrm{~h}$.

Human liver tissue sections. Fibrotic human liver tissue was collected from patients undergoing liver transplantation surgery at the Queen Elizabeth Hospital in Birmingham for end-stage liver diseases including non-alcoholic steatohepatitis (NASH), primary biliary cholangitis (PBC) and alcohol related liver disease (ALD). Non-fibrotic tissues were used as control. All tissues were accessed with informed written patient consent and research ethics committee approval provided by the Human Biomaterials Resource Centre at the University of Birmingham, UK. Tissue was formalin fixed upon collection from patients and paraffin embedded for immunohistochemical analysis.

Mice and breeding. A Cre/LoxP strategy was used to develop a constitutive EC-specific heterozygous deletion of $\mathrm{Erg}$ using Tie2-Cre-Erg $g^{f /+}\left(\mathrm{Erg}^{c E C-H e t}\right)^{23}$ or an inducible homozygous deletion of $\mathrm{Erg}$ using Pdgfb-iCreER-eGFP/Erg $f / f l$ mice $\left(E r g^{i E C-K O}\right)$. All experiments with $E r g$-deficient mice were conducted with age and gender matched animals at Imperial College London in accordance with the UK Animals (Scientific Procedures) act of 1986. All animals used were retained on a C57BL/6 background. Both male and female mice were used for experiments and were 8-10-weeks old. All experiments were conducted using littermate controls denoted in the text as Ergfl/fl.

Isolation of mouse lung endothelial cells. Primary mouse lung endothelial cells were isolated from the lungs of control $\mathrm{Erg}^{f l f l}$ and $\mathrm{Erg}^{\mathrm{cEC}-h e t}$ mice. Lungs were dissociated using GentleMACS C tubes and GentleMACS Dissociator (Miltenyi Biotec), digested with $0.1 \%$ collagenase type I (Invitrogen), and sieved through a 70 $\mu \mathrm{m}$-pore cell strainer (BD Falcon). Magnetic immunosorting (Dynabeads; Invitrogen) was used to select EC by a negative sort for Fc $\gamma$ RII/III receptor-positive macrophages followed by a positive sort for CD31-positive endothelial cells. Cells were plated in flasks precoated with a mixture of $0.1 \%$ gelatin (Sigma) and human plasma fibronectin (Chemicon) and cultured with EGM-2 media (Lonza).

\section{Carbon Tetrachloride $\mathrm{CCL}_{4}$ liver injury model. C57BL/6 J mice were obtained} from Harlan OLAC LTD or from in-house colonies. Acute hepatic inflammation was induced using intraperitoneal injections of $\mathrm{CCl}_{4}$ (carbon tetrachloride) (SigmaAldrich). $\mathrm{CCl}_{4}$ was diluted 1:4 with mineral oil, and injected intraperitoneally (i.p) at a concentration of $1 \mathrm{ml} \mathrm{kg}^{-1}$ (control animals were treated with i.p mineral oil alone). For chronic models, mice were injected with $\mathrm{CCl}_{4} \mathrm{IP}$ bi-weekly for 8 weeks. Indicated mice were allowed to recover for a further 3 weeks before the livers were collected. Experiments requiring TNF- $\alpha$ blockade were conducted using etanercept $\left(10 \mathrm{mg} \mathrm{kg}^{-1}\right)$ (Amgen). Mice were injected with etanercept intravenously (i.v.) 30 min prior to single $\mathrm{CCl}_{4}$ administration, control animals were given PBS alone and liver tissues collected $48 \mathrm{~h}$ post-injection. All $\mathrm{CCL}_{4}$ experiments were conducted at University of Birmingham with experimental procedures undertaken in 6-8-week-old mice and used under procedure in accordance the Animals (Scientific Procedures) Act of 1986.

Immunofluorescence analysis of tissue and HUVEC. Tissues were fixed in $4 \%$ paraformaldehyde for $2 \mathrm{~h}$ at room temperature, then transferred to $70 \%$ ethanol prior to being embedded in paraffin. Sections were de-paraffinised with Histoclear, re-hydrated for hematoxylin and eosin and picro-sirius red or heated to $95^{\circ} \mathrm{C}$ for 10 min with DAKO antigen retrieval solution for immunofluorescence staining. All sections were blocked with $2 \%$ BSA prior to incubation with primary antibodies (listed in Supplementary Table 2).

HUVEC for immunofluorescence and proximity ligation assay (PLA) were grown on gelatinised coverslips in 12 -well plates. Cells were fixed with $4 \%$ paraformaldehyde for $15 \mathrm{~min}$ prior to blocking with 3\% BSA for $1 \mathrm{~h}$. PLA was performed by manufacturer's instructions (Duolink Sigma). Primary antibodies listed in Supplementary Table 2. All tissue sections and HUVEC were mounted with Prolong Diamond (Molecular Probes) and allowed to dry overnight before imaging. Confocal microscopy was carried out on a Carl Zeiss LSM780. Images were analysed with ImageJ (NIH) and Volocity software (PerkinElmer).

Immunoblotting analysis. CelLytic reagent (Sigma), supplemented with $1 \mathrm{mM}$ phenylmethylsufony fluoride (PMSF) protease inhibitor cocktail and phosphatase inhibitor cocktail 2 and 3 (Sigma), was used to obtain whole cell protein lysates. Immunoblots were probed with the primary antibodies detailed in Supplementary Table 2. Primary antibodies were detected using fluorescently labelled secondary antibodies: goat $\alpha$-rabbit IgG DyLight 680 and goat $\alpha$-mouse IgG Dylight 800 (Thermo Scientific). Odyssey CLx imaging system (LI-COR Biosciences) and Odyssey 2.1 software was used for detection and quantification of fluorescence intensity. HRP-conjugated secondary antibodies were used in some instances for chemiluminescence detection and protein levels were quantified by densitometry and normalised against loading controls. Supplementary Fig. 12 for the uncropped immunoblots.

Immunoprecipitation (Co-IP). HUVECs or HSEC were treated with TGF $\beta 2$ (Human TGF- $\beta$ 2; Peprotech) $10 \mathrm{ng} \mathrm{ml}^{-1}$ for $30 \mathrm{~min}$ or SB-431542 (Abcam) $10 \mu \mathrm{gg}$ $\mathrm{ml}^{-1}$ for $1 \mathrm{~h}$ after $4 \mathrm{~h}$ serum starvation. Cells were collected in lysis buffer $(50 \mathrm{mM}$ Tris, pH 7.5, $150 \mathrm{mM} \mathrm{NaCl}, 0.1 \%$ Igepal, $1 \mathrm{mM}$ EDTA, $0.25 \%$ sodium deoxycholate) $^{37}$ supplemented with $1 \mathrm{mM}$ phenylmethylsufony fluoride (PMSF), protease inhibitor cocktail, and phosphatase inhibitor cocktail 2 and 3 (Sigma). Endonuclease treatment was conducted with the addition of 500 units of Benzonase (Sigma-Aldrich, UK) for $1 \mathrm{~h}$ at room temperature. After clarification by centrifugation at $4500 \times g, 1 \mathrm{mg}$ of total protein cell lysate was incubated with $3 \mu \mathrm{g}$ of $\alpha$-ERG antibody (sc-376293, Santa Cruz) or $3 \mu$ g of $\alpha$-mouse IgG (eBioscience) and Protein A/G PLUS-Agarose (sc-2003, Santa Cruz Biotechnology) overnight at $4{ }^{\circ} \mathrm{C}$. Beads with the immune-precipitated complex were washed with lysis buffer three times prior to denaturing in Laemmli buffer at $95^{\circ} \mathrm{C}$ for $5 \mathrm{~min}$. Samples were loaded on $12 \%$ acrylamide gels, transferred to nitrocellulose membranes and probed with $\alpha$-ERG (ab133264, Abcam) and $\alpha$ - Phospho SMAD3 (ab52903, Abcam), $\alpha$-SMAD3 (9523-S, Cell signalling), $\alpha$-SMAD2 (86F7, Cell signalling), $\alpha$-SMAD1 (9743 S, Cell Signalling) antibodies. HRP-conjugated secondary antibodies were used for chemiluminescence detection and protein levels were quantified by densitometry and normalised against loading controls. See Supplementary Fig. 13 for the uncropped immunoblots.

Reporter assays. Reporter assays in HUVEC were performed with the DualLuciferase Reporter Assay System (Promega) and a GloMax-Multi + Microplate Multimode Reader (Promega). Twenty-four hours after co-transfection with the luciferase reporters and expression plasmids and $18 \mathrm{~h}$ after stimulation with human recombinant BMP9 $\left(1 \mathrm{ng} \mathrm{ml}^{-1}\right.$, Peprotech) or TGF $\beta 2$ (10 $\mathrm{ng} \mathrm{ml}^{-1}$, Peprotech), HUVEC were lysed and reporter assays were performed in triplicate. For siRNA experiments, cells were transfected with $20 \mathrm{nM}$ ERG or control siRNAs and after $24 \mathrm{~h}$ transfected with the luciferase reporters and activated with the ligands. The ratio of luciferase signal to Renilla signal from each transfection was determined to control for well-to-well variation in transfection efficiency.

Real-time polymerase chain reaction. RNA was extracted from tissues and HUVEC using the RNeasy kit (Qiagen). Supercript III Reverse Transcriptase (Invitrogen) was used for first strand cDNA synthesis. Quantitative real-time PCR was carried out using PerfeCTa SYBR Green Fastmix (Quanta Biosciences) on a

Fig. 6 Etanercept prevents ERG loss during murine liver injury. a C57/B6 mice were injected with mineral oil (vehicle; bi-weekly i.p) or with chronic CCl administration (8 weeks, bi-weekly i.p); a third group was subjected to a 3-weeks recovery period following $\mathrm{CCL}_{4}$ administration. Representative immunofluorescence of liver tissue samples stained for VWF (grey scale, green in merge), SMA (grey scale, red in merge) and ERG (white). Scale bar 50 $\mu \mathrm{m}$. Quantification of $\mathbf{b}$ SMA expression and $\mathbf{c}$ ERG expression (three fields per mouse, $n=3$ ). Data were compared to mineral oil controls ( ${ }^{\star}$ ) or to chronic $\mathrm{CCL}_{4}$ administration (\#) by one-way ANOVA and Bonferroni multiple comparison post-test. $\mathbf{d}$ Representative immunofluorescence following acute (48 $\mathrm{h}$ ) mineral oil, $\mathrm{CCL}_{4}$ or co-administration of etanercept and $\mathrm{CCl}_{4}$. Staining for ERG (white); merged images showing VWF (green), SMA (red) and DAPI (blue). e pSMAD3 (white; stars); other colours as in d; scale bar $20 \mu \mathrm{m}$. $\mathbf{f}-\mathbf{h}$ : Quantification of $\mathbf{f}$ SMA, $\mathbf{g}$ ERG and $\mathbf{h}$ pSMAD3 expression (three fields per mouse, $n=3$ ). Data were compared to mineral oil controls $\left(^{*}\right)$ or to $48 \mathrm{~h} \mathrm{CCL}_{4}$ administration (\#) by one-way ANOVA and Bonferroni multiple comparison post-test. i Co-administration of etanercept and $\mathrm{CCL}_{4}$ for $48 \mathrm{~h}$ was able to significantly reduce SMA expression in Erg ${ }^{f l / f l}$ mice while it was ineffective in Erg ${ }^{C E C-H e t}$ mice. Representative immunofluorescence of liver tissue, colour scheme as above. Scale bar $50 \mu \mathrm{m}$. j Quantification of SMAD2/3 target gene expression by qPCR in liver tissue of Erg ${ }^{f l / f l}$ mice injected with etanercept and $\mathrm{CCL}_{4}$ compared to $\mathrm{CCL}_{4}$ alone $\left(^{\star}\right)$ or Erg ${ }^{c E C-H e t}$ treated with etanercept and $\mathrm{CCL}_{4}$ compared to $\mathrm{CCL}_{4}$ alone (\#). $\mathbf{k}$ Quantification of SMA expression (three fields per mouse, $n=3$ ). Data analysed by one-way ANOVA and Bonferroni multiple comparison post-test. All graphical data are mean \pm s.e.m., ${ }^{\star}$ or ${ }^{\#} P<0.05$, ${ }^{\star \star}$ or ${ }^{\# \#} P<0.01$, ${ }^{\star \star \star}$ or ${ }^{\# \# \#} P<0.001$ 
a Non-fibrotic

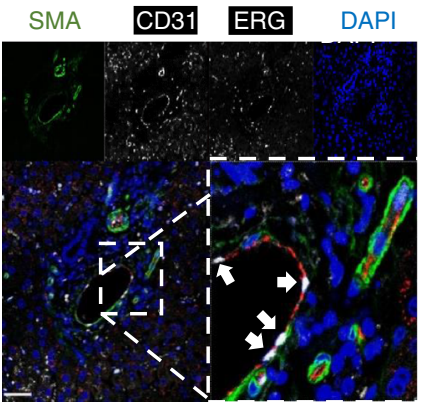

SMA/CD31/ERG/DAPI

ALD

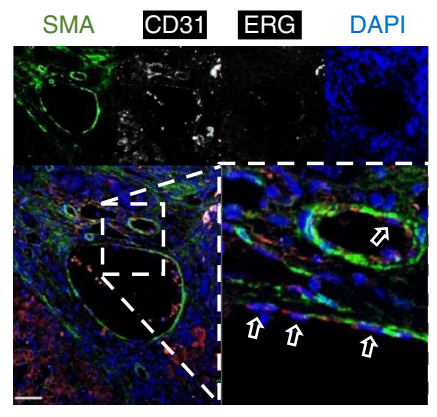

SMA/CD31/ERG/DAPI

C Non-fibrotic

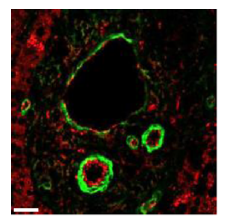

NASH SMA/CD31
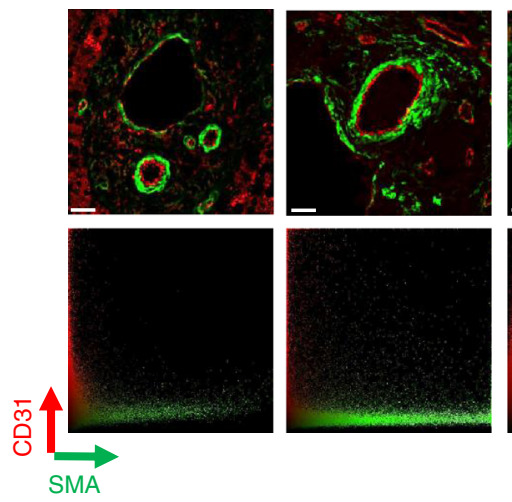

$\mathrm{NASH}$

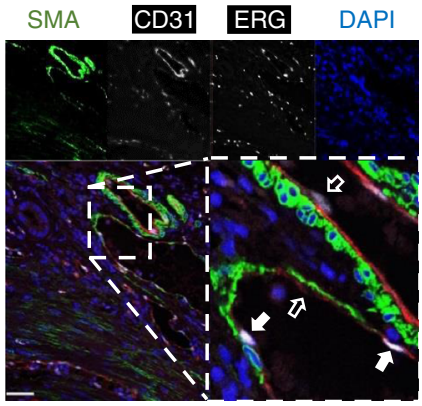

SMA/CD31/ERG/DAPI

PBC

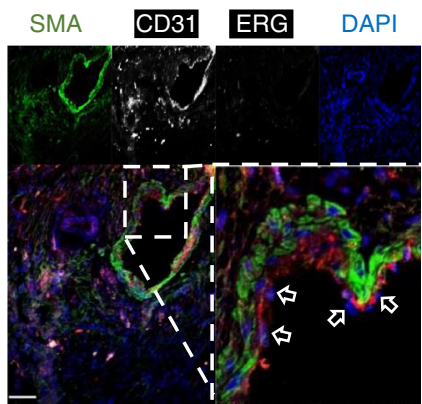

SMA/CD31/ERG/DAPI

d
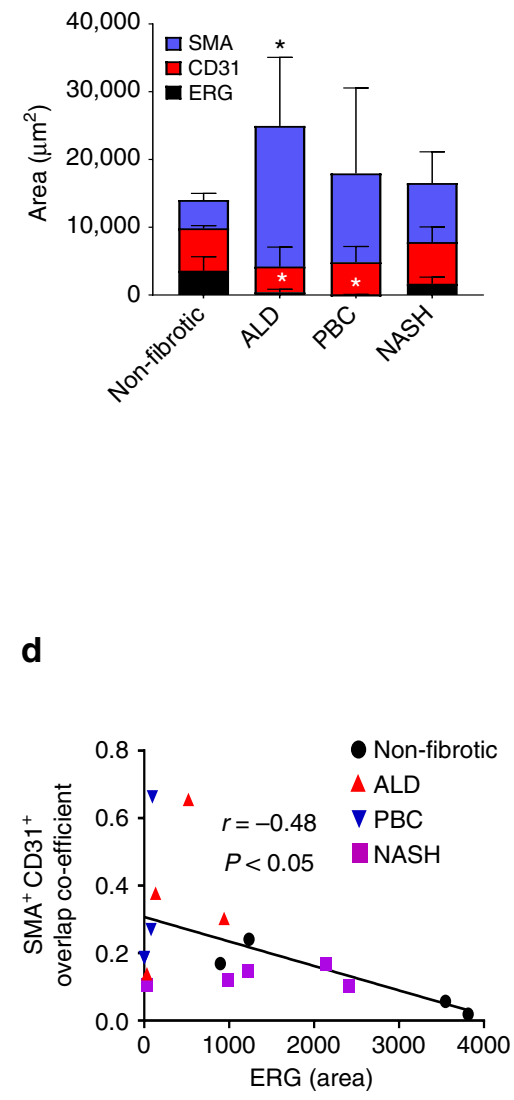

Fig. 7 Loss of ERG expression correlates with EndMT in end-stage human liver disease. a Representative immunofluorescence of human liver sections stained for SMA (green), CD31 (grey scale; red in merge), ERG (white) and DAPI (blue) were captured from non-fibrotic donor patients with chronic endstage NASH, PBC and ALD with enlarged merge panel. Filled white arrows identify $C D 31^{+} E R G^{+} E C$ whereas empty white arrows show $C D 31^{+} E R G^{-} E C$ (Scale bar $50 \mu \mathrm{m}$ ). b Quantification of SMA, CD31 and ERG expression (five fields per section, $n=4-5$ ). Scale bar $20 \mu \mathrm{m}$. Data were compared to non-fibrotic group $\left(^{*}\right)$ by one-way ANOVA and Dunnett's comparison post-test. c Representative images for CD31 (Red) and SMA (green) staining, showing co-localisation indicative of EndMT (arrows) and their pixel co-localisation profile in ALD, PBC and NASH patient samples. d Quantification of pixel co-localisation of CD31 (Red) and SMA (green) by overlap co-efficient was correlated with ERG expression for all individual human section data, non-fibrotic (black circles), NASH (purple squares), PBC (blue triangle down) and ALD (red triangle up). Correlation was calculated by linear regression, resulting in a significant negative correlation. All graphical data are mean \pm s.e.m., ${ }^{\star} P<0.05$

Bio-Rad CFX96 system. Oligonucleotides used for human and mouse are listed in Supplementary Tables 3, 4, respectively.

ChIP-qPCR. Chromatin immunoprecipitation (ChIP) was carried out using ChIPIT express kit (Active Motif). HUVEC were transfected with ERG or control siRNA $(20 \mathrm{nM}$, for $48 \mathrm{~h})$ or treated with PBS or TGF $2\left(10 \mathrm{ng} \mathrm{ml}^{-1}\right.$, for $\left.30 \mathrm{~min}\right)$, and cross-linked for $10 \mathrm{~min}$ with formaldehyde (final concentration of 1\%). Chromatin was sheared using a Bioruptor UCD-200 ultrasound sonicator (Diagenode), resulting in DNA fragments of 500-1000 bp in size. Chromatin was immunoprecipitated with $2 \mu \mathrm{g}$ antibody to ERG (sc-354X, Santa Cruz Biotechnology), SMAD3 (\#9523, Cell Signalling) or negative control rabbit IgG (PP64, Chemicon, Millipore) using protein $\mathrm{G}$ magnetic beads (Active Motif). Immunoprecipitated DNA was then used as template for qPCR using primers specific for genomic loci. Oligonucleotide sequences are listed in Supplementary Table 5.

Bioinformatics analysis. Gene ontology analysis was performed using Database for Annotation, Visualisation, and Integrated Discovery (DAVID), to identify overrepresented gene ontology categories from microarray data obtained from control and ERG-deficient HUVEC ${ }^{24}$. Functional clustering tool within DAVID assigned group gene associated with TGF signalling with an enrichment score of 1.3 (which corresponds to $P<0.05$ ). Microarray data are deposited in GEO database: GSE32984 24 . 
The JASPAR database (http://jaspar.genereg.net) was used to identify ERG transcription factor DNA binding motifs. Data obtained from genome-wide ChIPSeq for H3K27ac, H3K4Me3 and H3K4Mel histone modifications and RNA polymerase II occupancy in HUVEC and phyloP sequence conservation (conservation scores between -5 and +5 ) were based on Multiz alignment analysis of 100 vertebrate species (derived from the Broad Institute and publicly available from the ENCODE Consortium). Each track was visualised using the UCSC Genome Browser database (https://genome.ucsc.edu/index.html).

Statistical analysis. Data shown are representative of at least three experiment (unless otherwise stated) and are expressed as the mean \pm standard error of the mean (s.e.m.). For in vivo experiments group sizes were determined using estimates of variance and minimum detectable differences between groups that were based on our experience of characterising murine phenotypes. Animals were randomized using an identification numbers, allowing investigators to perform histological analyses blinded to animal genotype and treatment group. Statistical significance was determined by Student's unpaired $t$-test assuming unequal variances, using Prism 6.0 (Graph Pad). Differences were considered significant with a $P$-value $<0.05$

Data availability. The data supporting the findings of this study are available within the article and its Supplementary Information files and can also be obtained from the corresponding author upon reasonable request.

Received: 6 October 2016 Accepted: 24 August 2017

Published online: 12 October 2017

\section{References}

1. Craig, R. \& Shelton, N. Health Survey for England-2007: Healthy lifestyles: knowledge, attitudes and behaviour. Ref Type: Online Source. (2008).

2. Liu, T., Zhou, Y., Ko, K. S. \& Yang, H. Interactions between myc and mediators of inflammation in chronic liver diseases. Mediators Inflamm. 2015, 276850 (2015).

3. Lee, Y. A., Wallace, M. C. \& Friedman, S. L. Pathobiology of liver fibrosis: a translational success story. Gut. 64, 830-841 (2015).

4. Iwakiri, Y., Shah, V. \& Rockey, D. C. Vascular pathobiology in chronic liver disease and cirrhosis-current status and future directions. J. Hepatol. 61, 912-924 (2014).

5. Chen, W. X. et al. Quantitative analysis of transforming growth factor beta 1 mRNA in patients with alcoholic liver disease. World J. Gastroenterol. 8, 379-381 (2002).

6. Ding, B. S. et al. Divergent angiocrine signals from vascular niche balance liver regeneration and fibrosis. Nature 505, 97-102 (2014).

7. DeLeve, L. D. Liver sinusoidal endothelial cells and liver regeneration. J. Clin. Invest. 123, 1861-1866 (2013).

8. $\mathrm{Hu}$, J. et al. Endothelial cell-derived angiopoietin-2 controls liver regeneration as a spatiotemporal rheostat. Science 343, 416-419 (2014).

9. Zeisberg, E. M. et al. Endothelial-to-mesenchymal transition contributes to cardiac fibrosis. Nat. Med. 13, 952-961 (2007)

10. Cooley, B. C. et al. TGF-beta signaling mediates endothelial-to-mesenchymal transition (EndMT) during vein graft remodeling. Sci. Transl. Med. 6, 227ra34 (2014).

11. Chen, P. Y. et al. Endothelial-to-mesenchymal transition drives atherosclerosis progression. J. Clin. Invest. 125, 4514-4528 (2015).

12. Ten, D. P., Egorova, A. D., Goumans, M. J., Poelmann, R. E. \& Hierck, B. P. TGF-beta signaling in endothelial-to-mesenchymal transition: the role of shear stress and primary cilia. Sci. Signal. 5, t2 (2012).

13. Chen, P. Y. et al. FGF regulates TGF-beta signaling and endothelial-tomesenchymal transition via control of let-7 miRNA expression. Cell Rep. 2, 1684-1696 (2012)

14. Mahler, G. J., Farrar, E. J. \& Butcher, J. T. Inflammatory cytokines promote mesenchymal transformation in embryonic and adult valve endothelial cells. Arterioscler. Thromb. Vasc. Biol. 33, 121-130 (2013).

15. Krenning, G., Barauna, V. G., Krieger, J. E., Harmsen, M. C. \& Moonen, J. R. Endothelial plasticity: shifting phenotypes through force feedback. Stem Cells Int. 2016, 9762959 (2016).

16. Goumans, M. J., Liu, Z. \& Ten, D. P. TGF-beta signaling in vascular biology and dysfunction. Cell Res. 19, 116-127 (2009).

17. Nolan-Stevaux, O. et al. Endoglin requirement for BMP9 signaling in endothelial cells reveals new mechanism of action for selective anti-endoglin antibodies. PLoS ONE 7, e50920 (2012).

18. Morikawa, M., Koinuma, D., Miyazono, K. \& Heldin, C. H. Genome-wide mechanisms of Smad binding. Oncogene 32, 1609-1615 (2013).

19. Goumans, M. J. et al. Balancing the activation state of the endothelium via two distinct TGF-beta type I receptors. EMBO J. 21, 1743-1753 (2002).
20. Goumans, M. J. et al. Activin receptor-like kinase (ALK)1 is an antagonistic mediator of lateral TGFbeta/ALK5 signaling. Mol. Cell 12, 817-828 (2003).

21. Shah, A. V., Birdsey, G. M. \& Randi, A. M. Regulation of endothelial homeostasis, vascular development and angiogenesis by the transcription factor ERG. Vascul. Pharmacol. 86, 3-13 (2016).

22. Ginsberg, M. et al. Efficient direct reprogramming of mature amniotic cells into endothelial cells by ETS factors and TGFbeta suppression. Cell 151, 559-575 (2012).

23. Birdsey, G. M. et al. The endothelial transcription factor ERG promotes vascular stability and growth through Wnt/beta-catenin signaling. Dev. Cell 32, 82-96 (2015).

24. Yuan, L. et al. RhoJ is an endothelial cell-restricted Rho GTPase that mediates vascular morphogenesis and is regulated by the transcription factor ERG. Blood 118, 1145-1153 (2011)

25. Birdsey, G. M. et al. The transcription factor Erg regulates expression of histone deacetylase 6 and multiple pathways involved in endothelial cell migration and angiogenesis. Blood 119, 894-903 (2012).

26. Sperone, A. et al. The transcription factor Erg inhibits vascular inflammation by repressing NF-kappaB activation and proinflammatory gene expression in endothelial cells. Arterioscler. Thromb. Vasc. Biol. 31, 142-150 (2011).

27. Yuan, L. et al. Antiinflammatory effects of the ETS factor ERG in endothelia cells are mediated through transcriptional repression of the interleukin-8 gene. Circ. Res. 104, 1049-1057 (2009).

28. Dryden, N. H. et al. The transcription factor Erg controls endothelial cell quiescence by repressing activity of nuclear factor (NF)-kappaB p65. J. Biol. Chem. 287, 12331-12342 (2012).

29. Korchynskyi, O. \& Ten, D. P. Identification and functional characterization of distinct critically important bone morphogenetic protein-specific response elements in the Id1 promoter. J. Biol. Chem. 277, 4883-4891 (2002).

30. Zawel, L. et al. Human Smad3 and Smad4 are sequence-specific transcription activators. Mol. Cell 1, 611-617 (1998).

31. Medici, D. et al. Conversion of vascular endothelial cells into multipotent stemlike cells. Nat. Med. 16, 1400-1406 (2010).

32. Xiao, L. et al. Tumor endothelial cells with distinct patterns of TGFbeta-driven endothelial-to-mesenchymal transition. Cancer Res. 75, 1244-1254 (2015).

33. Pimanda, J. E. et al. Endoglin expression in the endothelium is regulated by Fli-1, Erg, and Elf-1 acting on the promoter and a -8-kb enhancer. Blood 107, 4737-4745 (2006).

34. Harrison, R. E. et al. Molecular and functional analysis identifies ALK-1 as the predominant cause of pulmonary hypertension related to hereditary haemorrhagic telangiectasia. J. Med. Genet. 40, 865-871 (2003).

35. Trembath, R. C. et al. Clinical and molecular genetic features of pulmonary hypertension in patients with hereditary hemorrhagic telangiectasia. N. Engl. J. Med. 345, 325-334 (2001).

36. Cox, M. K., Appelboom, B. L., Ban, G. I. \& Serra, R. Erg cooperates with TGF-beta to control mesenchymal differentiation. Exp. Cell Res. 328, 410-418 (2014).

37. Fang, J. et al. Ets related gene and smad 3 proteins collaborate to activate transforming growth factor-beta mediated signaling pathway in ETS related gene-positive prostate cancer cells. J. Pharm. Sci. Pharmacol. 1, 175-181 (2014).

38. Koinuma, D. et al. Chromatin immunoprecipitation on microarray analysis of Smad2/3 binding sites reveals roles of ETS1 and TFAP2A in transforming growth factor beta signaling. Mol. Cell Biol. 29, 172-186 (2009).

39. Vijayaraj, P. et al. Erg is a crucial regulator of endocardial-mesenchymal transformation during cardiac valve morphogenesis. Development 139, 3973-3985 (2012).

40. McClain, C. J., Hill, D. B., Song, Z., Deaciuc, I. \& Barve, S. Monocyte activation in alcoholic liver disease. Alcohol 27, 53-61 (2002).

41. Gordon, M. A. et al. Primary biliary cirrhosis shows association with genetic polymorphism of tumour necrosis factor alpha promoter region. J. Hepatol. 31, 242-247 (1999)

42. Sato, A. et al. Involvement of the TNF and FasL produced by CD11b Kupffer cells/macrophages in CCl4-induced acute hepatic injury. PLoS ONE 9, e92515 (2014).

43. Sehitoglu, I. et al. Protective effect of infliximab against carbon tetrachlorideinduced hepatotoxicity. J. Environ. Pathol. Toxicol. Oncol. 34, 175-182 (2015)

44. Kovacs, A. et al. Successful etanercept treatment for primary biliary cirrhosis associated with rheumatoid arthritis. Isr. Med. Assoc. J. 17, 114-116 (2015).

45. He, T. C., Chan, T. A., Vogelstein, B. \& Kinzler, K. W. PPARdelta is an APCregulated target of nonsteroidal anti-inflammatory drugs. Cell 99, 335-345 (1999).

\section{Acknowledgements}

Prof. S. Hubscher (University of Birmingham, UK) and Prof D. Haskard (Imperial College London, UK) for helpful discussions. Prof. H. Gerhardt (Max-Delbrück Center for Molecular Medicine, Berlin, Germany) for generation of the Pdgfb-iCreER-eGFP/ 
$\operatorname{Erg}^{f l / f l}$ mice $\left(E r g^{i E C-K O}\right)$. The NIHR Biomedical Research Unit in Liver Disease at Birmingham University for supporting this work. This work was funded by grants from the British Heart Foundation (PG/09/096 \& RG/11/17/29256). The Facility for Imaging by Light Microscopy (FILM) at Imperial College London is part supported by funding from the Wellcome Trust (grant 104931/Z/14/Z) and BBSRC (grant BB/L015129/1).

\section{Author contributions}

N.P.D. conceived and designed and carried out in vitro and in vivo experiments, analysed and conceptualised results, wrote the manuscript. C.R.P. designed and performed experiments, analysed and interpreted results. C.R. and L.O.A. performed experiments and analysed results. Y.Y. and V.K. performed bioinfomatic analysis; A.C. and G.W performed experiments and analysed results; G.M.B., J.C.M. and D.H.A. contributed to scientific discussion; P.L. provided reagents, advice and contributed to scientific discussion; A.M.R. provided funding, conceived, designed and supervised the study, interpreted results and wrote the manuscript.

\section{Additional information}

Supplementary Information accompanies this paper at doi:10.1038/s41467-017-01169-0.

Competing interests: The authors declare no competing financial interests.

Reprints and permission information is available online at http://npg.nature.com/ reprintsandpermissions/

Publisher's note: Springer Nature remains neutral with regard to jurisdictional claims in published maps and institutional affiliations.

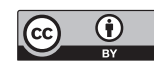
adaptation, distribution and reproduction in any medium or format, as long as you give appropriate credit to the original author(s) and the source, provide a link to the Creative Commons license, and indicate if changes were made. The images or other third party material in this article are included in the article's Creative Commons license, unless indicated otherwise in a credit line to the material. If material is not included in the article's Creative Commons license and your intended use is not permitted by statutory regulation or exceeds the permitted use, you will need to obtain permission directly from the copyright holder. To view a copy of this license, visit http://creativecommons.org/ licenses/by/4.0/.

(C) The Author(s) 2017 